\title{
A partitioned Newton method for the interaction of a fluid and a 3D shell structure
}

\author{
Miguel Ángel Fernández* — Jean-Frédéric Gerbeau* \\ Antoine Gloria** — Marina Vidrascu* \\ * INRIA, Paris-Rocquencourt, BP 105, F-78153 Le Chesnay cedex \\ ** INRIA, Lille-Nord Europe, 40 avenue Halley, F-59650 Villeneuve d'Ascq
}

\begin{abstract}
We propose a new fluid-structure algorithm based on a domain decomposition paradigm. The method is based on the principle "linearize first, then decompose" whereas the usual schemes are generally "nonlinear in subdomains". The proposed approach is more attractive when the complexity of the structure is high, which is the case with the structural model used in this study (nonlinear $3 D$ shell). Another contribution of this paper is to investigate the use of a Neumann-Neumann preconditioner for the linearized problem. In particular, it is shown that when this preconditioner is adequately balanced, it tends to the Dirichlet-Neumann preconditioner because of the heterogeneity of the fluid-structure problem.

RÉSUMÉ. Nous proposons un nouvel algorithme fluide-structure inspiré par une technique de décomposition de domaine. La méthode est basée sur le principe "linéariser puis décomposer » alors que les algorithmes existants sont généralement "non linéaires par sousdomaine ». L'approche proposée est d'autant plus attractive que la complexité de la structure est grande, ce qui est le cas avec le modèle de structure utilisé dans cette étude (coque $3 D$ non linéaire). Une autre contribution de cet article concerne l'utilisation du préconditionneur Neumann-Neumann pour le problème linéarisé. On montre en particulier que lorsque ce préconditionneur est correctement équilibré, il tend vers un préconditionneur DirichletNeumann, du fait de l'hétérogénéité des problèmes fluide et structure.

KEYWORDS: fluid-structure interaction, $3 D$ shell finite elements, domain decomposition, partitioned schemes, Newton algorithm.

MOTS-CLÉS : interaction fluide-structure, éléments de coque 3D, décomposition de domaine, schémas partitionnés, algorithme de Newton.
\end{abstract}

DOI:10.3166/EJCM.19.479-512 @ 2010 Lavoisier, Paris

EJCM - 19/2010. Fluid-structure interaction, pages 479 to 512 


\section{Introduction}

Many works devoted to fluid-structure interaction algorithms have been inspired by domain decomposition techniques. Usually the proposed methods have been devised following the rule "apply domain decomposition to the nonlinear global problem and then solve on each subdomain the nonlinear problems". On the contrary, in other fields - for example nonlinear elasticity (Le Tallec, 1994) - domain decomposition is usually applied with the rule "linearize first, then solve the tangent problem using domain decomposition". The purpose of this paper is to propose a fluid-structure algorithm based on the last rule.

Fluid-structure algorithms are too numerous to be reviewed exhaustively. A classification of the various approaches is not obvious either. To begin with, we can consider two groups of methods: the "strongly coupled" and the "loosely coupled" schemes. This distinction is quite clear since it corresponds to a precise property: those schemes which can ensure a well-balanced energy transfer between the fluid and the structure can be called "strongly coupled", the other ones are "loosely coupled". All the methods presented in this study are strongly coupled. Loosely coupled schemes, which are very powerful in many applications but can be unstable in others, are not considered here. We refer, for example, to (Piperno et al., 1995; Felippa et al., 2001; Farhat et al., 2006) for explicit coupling schemes used in aeroelasticity and to (Guidoboni et al., 2009; Burman et al., 2009; Fernández et al., 2007; Quaini et al., 2007) for explicit and semi-implicit schemes recently proposed for blood flows. Explanations of the numerical difficulties encountered for incompressible flows and in presence of strong added-mass effect can be found in (Causin et al., 2005; Förster et al., 2006; van Brummelen, 2009).

We can then distinguish "monolithic" and "partitioned" schemes. For example, an ad hoc solver whose purpose is to solve simultaneously the fluid and the structure typically leads to a monolithic scheme (see (Rugonyi et al., 2001; Tezduyar, 2001; Zhang et al., 2003; Heil, 2004; Bathe et al., 2004; Hübner et al., 2004; Bazilevs et al., 2006; Badia et al., 2008b; Küttler et al., 2009; Gee et al., 2010), for instance). When the fluid and the structure software are distinct, the scheme is said to be partitioned. In fluid-structure interaction this kind of schemes dates back at least to (Park et al., 1977; Felippa et al., 1980). Partitioned scheme can be strongly coupled as soon as sub-iterations are performed at every time step. The number of subiterations being very large in some applications, acceleration techniques have been investigated in several articles: for example a steepest descent approach is proposed in (Le Tallec et al., 2001), an Aitken acceleration based on the two previously computed solutions is used in (Mok et al., 2001b; Küttler et al., 2008), a least-square method which uses several previously computed solutions is proposed in (Vierendeels, 2006). Let us also mention the modified-mass method (Tezduyar et al., 2006), the interface-GMRES method (Michler et al., 2005; Michler et al., 2006) and the partitioning-based multigrid methods (Hron et al., 2006; van Brummelen et al., 2008). 
It is well-known, in particular since (Le Tallec et al., 2001) and more recently (Deparis et al., 2004; Deparis et al., 2006), that fluid-structure problems can be tackled with domain decomposition approaches. Indeed, a fluid-structure problem can be viewed as a general continuum mechanics problem set on one domain which is split into a fluid part and a structure part. The fluid-structure coupling conditions then appear as the transmission conditions which ensure that the solution of the global problem is obtained by "sticking" the two sub-problem solutions. This point of view has been adopted in various studies, either with the so-called "Dirichlet-Neumann" algorithms, see for example (Mok et al., 2001a; Gerbeau et al., 2003; Fernández et al., 2005), or with "Neumann-Neumann" algorithms (Deparis et al., 2004; Deparis et al., 2006).

The algorithm proposed in this paper can be viewed as a monolithic scheme in the sense that a Newton algorithm is applied to the global fluid-structure problem. But, it is more conform to the practical implementation to consider it as a partitioned scheme, since the fluid and the structure are solved with two different solvers, with their own schemes, and can be run in parallel. In contrast to the methods following the rule "decompose the nonlinear problem", these solvers are only used to solve the tangent problems and to evaluate nonlinear residuals. The use of two different solvers has well-known advantages (re-usability of existing codes, flexible choice of the numerical methods adapted to each sub-problem, etc.). In addition, our numerical experiments show that in some cases - for example when the structure is multilayered - the approach advocated in this study ("linearize first, then decompose") is actually more robust than the usual ones consisting of the iterative resolution of nonlinear problems.

We investigate Dirichlet-Neumann and Neumann-Neumann algorithms to solve the tangent problems. The Schur complements defining the Neumann-Neumann preconditioner are then balanced using state-of-the-art methods of structural mechanics. As a consequence of this balancing, we observe that, for the FSI problem at hand, the Neumann-Neumann preconditioner actually behaves as a Dirichlet-Neumann preconditioner. To our knowledge, this fact had not been noticed in previous studies about Neumann-Neumann algorithm for FSI problems.

The remainder of the paper is organized as follows. In Section 2 we review some standard approaches to solve fluid-structure interaction problems, in particular those based on domain decomposition arguments. In Section 3 we recall the fluid and solid models and we set the main notation. In Section 4 we propose a short review on constitutive laws that have been developed recently to model soft tissues, and in particular the arterial wall. The time scheme is presented in Section 5. In Section 6 the new algorithm is introduced. We propose in Section 6.3 a simplified complexity analysis to compare the efficiency of the proposed algorithm with other existing approaches. The conclusion may be sum up as follows: the more expensive the structure problem and nonlinear the fluid the more competitive is expected this new formulation. Numerical results and a comparison with existing methods are reported in Section 7. Finally, some conclusions are given in Section 8. 


\section{Classical solution methods}

In this section, we briefly review some of the existing algorithms for the numerical solution of the nonlinear system arising in the time discretization of the fluid-structure problem with an implicit coupling scheme. These methods are typically based on the application of a particular nonlinear iterative method to three different formulations of the nonlinear coupled system.

In general, the time discretization of a fluid-structure problem with an implicit coupling scheme leads to a coupled nonlinear problem of the type: Find the interface displacement $\gamma$, the fluid state $\boldsymbol{x}_{f}$ and the solid state $\boldsymbol{x}_{s}$ such that

$$
\text { Formulation (I): } \quad\left\{\begin{aligned}
\mathcal{F}\left(\boldsymbol{x}_{f}, \boldsymbol{\gamma}\right) & =0, \\
\mathcal{S}\left(\boldsymbol{x}_{s}, \boldsymbol{\gamma}\right) & =0, \\
\mathcal{I}\left(\boldsymbol{x}_{f}, \boldsymbol{x}_{s}\right) & =0 .
\end{aligned}\right.
$$

Equations $[1]_{1}$ and $[1]_{2}$ ensure the equilibrium of momentum when the fluid and the solid are subjected to an interface displacement $\gamma$, whereas the last equation enforces the equilibrium of mechanical stresses at the interface.

Problem [1] can be reformulated in terms of $\gamma$ by eliminating the fluid and solid unknowns $\boldsymbol{x}_{f}, \boldsymbol{x}_{s}$. This yields to the so-called Steklov-Poincaré formulation: Find the interface displacement $\gamma$ such that,

$$
\text { Formulation (II): } \quad S_{f}(\gamma)+S_{s}(\gamma)=0 \text {. }
$$

Here, $S_{f}$ and $S_{s}$ stand for the fluid and solid Steklov-Poincaré operators which can be defined as follows: for a given interface displacement $\gamma, S_{f}(\gamma)$ gives the stress exerted by the fluid on the interface, and analogously for $S_{s}$. This notation will be made precise below. In Section 5.2, we shall describe the link between [1] and [2].

Finally, the composition of [2] with the inverse operator $S_{s}^{-1}$ gives rise to the socalled Dirichlet-to-Neumann formulation:

$$
\text { Formulation (III): } \quad S_{s}^{-1}\left(-S_{f}(\gamma)\right)-\gamma=0 .
$$

Formally speaking, Formulations (II) and (III) are similar. Nevertheless, we prefer to distinguish them since they correspond to different approaches in the literature. The denominations "Dirichlet-Neumann formulation" and "Steklov-Poincaré formulation" are purely conventional (both of them clearly involve Steklov-Poincaré operators).

The three following paragraphs address a brief state-of-the-art on the iterative methods for the numerical solution of [1], [2] and [3]. 


\subsection{Dirichlet to Neumann formulations}

Formulation (III) reduces problem [1] to the determination of a fixed point of the Dirichlet-to-Neumann operator $\gamma \mapsto S_{s}^{-1}\left(-S_{f}(\gamma)\right)$. This motivates the use of fixedpoint based methods (Le Tallec et al., 2001; Nobile, 2001; Mok et al., 2001b; Mok et al., 1999):

$$
\gamma^{k+1}=\omega^{k} S_{s}^{-1}\left(-S_{f}\left(\gamma^{k}\right)\right)+\left(1-\omega^{k}\right) \gamma^{k}
$$

with $\omega^{k}$ a given relaxation parameter which is chosen in order to enhance convergence (Mok et al., 2001b; Mok et al., 1999; Deparis, 2004; Küttler et al., 2008). Alternatively, one can use Newton based methods (Gerbeau et al., 2003; Fernández et al., 2005) for a fast convergence towards the solution of [3]. This requires the solution of a tangent problem of the type

$$
\left(J\left(\gamma^{k}\right)-\boldsymbol{I}\right) \delta \gamma=-\left(S_{s}^{-1}\left(-S_{f}\left(\gamma^{k}\right)\right)-\gamma^{k}\right)
$$

where $J(\gamma)$ stands for the Jacobian, or approximated Jacobian (Gerbeau et al., 2003), of the composed operator $\gamma \mapsto S_{s}^{-1}\left(-S_{f}(\gamma)\right)$. It is worth noticing that exact Jacobian computations require shape derivative calculus for the fluid (Fernández et al., 2005) (see also (Dettmer et al., 2006; Bazilevs et al., 2008; van der Zee et al., 2010)). Let us also stress the fact that these methods are naturally partitioned.

\subsection{Symmetric Steklov-Poincaré formulation}

The Dirichlet-Neumann formulations share a common feature: their implementation is purely sequential. The Steklov-Poincaré formulation [2] may allow to set up parallel algorithms to solve the interface equation.

Following the presentation of Deparis et al. (Deparis et al., 2006), the nonlinear problem [2] can be solved through nonlinear Richardson iterations:

$$
P\left(\gamma^{k+1}-\gamma^{k}\right)=\omega^{k}\left(-S_{f}\left(\gamma^{k}\right)-S_{s}\left(\gamma^{k}\right)\right),
$$

for an appropriate choice of the preconditioner $P$, namely

$$
P_{k}^{-1}=\alpha^{k}\left[S_{f}^{\prime}\left(\gamma^{k}\right)\right]^{-1}+\left(1-\alpha^{k}\right)\left[S_{s}^{\prime}\left(\gamma^{k}\right)\right]^{-1},
$$

where $\boldsymbol{\lambda} \mapsto S_{f}^{\prime}(\beta) \cdot \boldsymbol{\lambda}$ is the differential of $S_{f}$ at $\beta$, and $\left[S_{f}^{\prime}(\beta)\right]^{-1}$ its inverse. This choice generalizes the standard preconditioners of linear domain decomposition methods (for which $S^{\prime}=S$ ). If $\alpha_{k}$ is 0,1 or 0.5 we retrieve, respectively, DirichletNeumann, Neumann-Dirichlet or Neumann-Neumann preconditioners. On the other hand, since equation [2] is nonlinear, one can apply a Newton method,

$$
\left(S_{f}^{\prime}\left(\gamma^{k}\right)+S_{s}^{\prime}\left(\gamma^{k}\right)\right)\left(\gamma^{k+1}-\gamma^{k}\right)=-S_{f}\left(\gamma^{k}\right)-S_{s}\left(\gamma^{k}\right),
$$


which corresponds to the nonlinear Richardson iteration [6] preconditioned with $P_{k}=$ $S_{f}^{\prime}\left(\gamma^{k}\right)+S_{s}^{\prime}\left(\gamma^{k}\right)$ and $\omega^{k}=1$. This linear equation can be solved, for example, by an operator-free GMRES algorithm, with or without preconditioning. For instance, in (Deparis et al., 2006) the authors propose to use the preconditioners [7].

The Newton method applied to the Dirichlet-Neumann formulation is not equivalent to the Newton method applied to the Steklov formulation, since the roles played by the fluid and by the structure are not symmetric in the first approach, whereas they are in the second. After linearization, one cannot compose [5] with $S_{s}$ to retrieve [8]. Finally [7] is not equivalent to [8] since in general $(A+B)^{-1} \neq A^{-1}+B^{-1}$.

The advantage of formulation (II) compared to formulation (III) is that the fluid and the structure sub-problems can be solved simultaneously and independently for the residual computation (right-hand sides of [6]) and the application of the preconditioner $\left(S_{f}^{\prime}\right.$ and $S_{s}^{\prime}$ ) as soon as $\alpha \notin\{0,1\}$. However, as we shall see in Section 6.3, a simplified complexity analysis shows that the overall computational costs of both methods might be of the same order, for instance, whenever the cost of the fluid subproblem solution is cheaper.

\subsection{Fully monolithic formulation}

A common approach in the numerical solution of nonlinear systems, arising in implicit coupling, consists in applying a Newton based algorithm to the global formulation [1]. This requires the repeated solution of a tangent (or approximated tangent) problem with the following block structure:

$\left[\begin{array}{ccc}\mathrm{D}_{\boldsymbol{x}_{f}} \mathcal{F}\left(\boldsymbol{x}_{f}, \boldsymbol{\gamma}\right) & 0 & \mathrm{D}_{\gamma} \mathcal{F}\left(\boldsymbol{x}_{f}, \boldsymbol{\gamma}\right) \\ 0 & \mathrm{D}_{\boldsymbol{x}_{s}} \mathcal{S}\left(\boldsymbol{x}_{s}, \boldsymbol{\gamma}\right) & \mathrm{D}_{\gamma} \mathcal{S}\left(\boldsymbol{x}_{s}, \boldsymbol{\gamma}\right) \\ \mathrm{D}_{\boldsymbol{x}_{f}} \mathcal{I}\left(\boldsymbol{x}_{f}, \boldsymbol{x}_{s}\right) & \mathrm{D}_{\boldsymbol{x}_{s}} \mathcal{I}\left(\boldsymbol{x}_{f}, \boldsymbol{x}_{s}\right) & 0\end{array}\right]\left[\begin{array}{c}\delta \boldsymbol{x}_{f} \\ \delta \boldsymbol{x}_{s} \\ \delta \gamma\end{array}\right]=-\left[\begin{array}{c}\mathcal{F}\left(\boldsymbol{x}_{f}, \boldsymbol{\gamma}\right) \\ \mathcal{S}\left(\boldsymbol{x}_{s}, \boldsymbol{\gamma}\right) \\ \mathcal{I}\left(\boldsymbol{x}_{f}, \boldsymbol{x}_{s}\right)\end{array}\right]$

Newton algorithms based on the numerical solution of [9] (or re-arrangements of it) in a monolithic fashion, i.e. using global direct or iterative methods, have been reported in (Tezduyar, 2001; Zhang et al., 2003; Heil, 2004; Bathe et al., 2004; Heil et al., 2008; Küttler et al., 2009; Gee et al., 2010). This way to solve [9] is not partitioned. Alternatively, system [9] can be solved in a partitioned manner through a block-Gauss elimination of $\delta \boldsymbol{x}_{f}$, which leads to the so called block-Newton methods (Matthies $e t$ al., 2002; Matthies et al., 2003).

The formulations recalled in Sections 2.1 and 2.2 are first based on the coupling conditions, giving rise to a nonlinear equation on the interface, which involves nonlinear sub-problems. The formulation is therefore partitioned at the nonlinear level (and therefore at the linear level too). The formulation recalled in Section 2.3 linearizes the full problem first, the associated linear problem being solved by a monolithic approach. This formulation is therefore monolithic at the nonlinear and linear levels. The algorithm we introduce in Section 6 turns out to combine both approaches: It first treats the nonlinearity of the whole problem through a Newton method (which makes 
it monolithic at the nonlinear level), and then it uses a Steklov-Poincaré formulation on the tangent problem (which makes it partitioned at the linear level).

\section{Mechanical setting}

Let $\widehat{\Omega}=\widehat{\Omega}_{f} \cup \widehat{\Omega}_{s}$ be a reference configuration of the system, see Figure 1. We introduce the motion of the solid medium

$$
\widehat{\varphi}_{s}: \widehat{\Omega}_{s} \times \mathbb{R}^{+} \longrightarrow \mathbb{R}^{3} .
$$

The current configuration of the structure is then denoted by

$$
\Omega_{s}(t)=\widehat{\varphi}_{s}\left(\widehat{\Omega}_{s}, t\right) .
$$

We introduce the deformation gradient $\widehat{\boldsymbol{F}}_{s}(\widehat{\boldsymbol{x}}, t) \stackrel{\text { def }}{=} \boldsymbol{\nabla}_{\widehat{\boldsymbol{x}}} \widehat{\boldsymbol{\varphi}}_{s}(\widehat{\boldsymbol{x}}, t)$, and its determinant $\widehat{J}_{s}(\widehat{\boldsymbol{x}}, t) \stackrel{\text { def }}{=} \operatorname{det} \widehat{\boldsymbol{F}}_{s}(\widehat{\boldsymbol{x}}, t)$. The displacement of the solid domain is given by $\widehat{\boldsymbol{d}_{s}}(\widehat{\boldsymbol{x}}, t) \stackrel{\text { def }}{=} \widehat{\boldsymbol{\varphi}}_{s}(\widehat{\boldsymbol{x}}, t)-\widehat{\boldsymbol{x}}$. The fluid domain $\Omega_{f}(t)$ is parametrized by the Arbitrary Lagrangian Eulerian ALE mapping (see (Donéa et al., 1982), for instance),

$$
\widehat{\mathcal{A}}: \widehat{\Omega}_{f} \times \mathbb{R}^{+} \longrightarrow \mathbb{R}^{3},
$$

such that $\Omega_{f}(t)=\widehat{\mathcal{A}}\left(\widehat{\Omega}_{f}, t\right)$. In the sequel we will use the notation $\widehat{\mathcal{A}}_{t} \stackrel{\text { def }}{=} \widehat{\mathcal{A}}(\cdot, t)$, and the superscript $\widehat{ }$ will be related to fields defined on the reference configuration $\widehat{\Omega}_{f}$ or $\widehat{\Omega}_{s}$. In addition, for a given Eulerian fluid quantity $q$ (i.e. defined in $\Omega_{f}(t)$ for $t>0$ ) we will denote its ALE description by $\widehat{q}$, as a field defined in $\widehat{\Omega}_{f} \times \mathbb{R}^{+}$as

$$
\widehat{q}(\widehat{\boldsymbol{x}}, t)=q\left(\widehat{\mathcal{A}}_{t}(\widehat{\boldsymbol{x}}), t\right), \quad \forall \widehat{\boldsymbol{x}} \in \widehat{\Omega}_{f} .
$$

We introduce the deformation gradient of the fluid domain

$$
\widehat{\boldsymbol{F}}_{f}(\widehat{\boldsymbol{x}}, t) \stackrel{\text { def }}{=} \nabla_{\widehat{x}} \widehat{\mathcal{A}}(\widehat{\boldsymbol{x}}, t),
$$

and its determinant $\widehat{J}_{f}(\widehat{\boldsymbol{x}}, t) \stackrel{\text { def }}{=} \operatorname{det} \widehat{\boldsymbol{F}}_{f}(\widehat{\boldsymbol{x}}, t)$. The displacement of the fluid domain is given by $\widehat{\boldsymbol{d}}_{f}(\widehat{\boldsymbol{x}}, t) \stackrel{\text { def }}{=} \widehat{\mathcal{A}}(\widehat{\boldsymbol{x}}, t)-\widehat{\boldsymbol{x}}$ and its velocity by

$$
\widehat{\boldsymbol{w}} \stackrel{\text { def }}{=} \frac{\partial \widehat{\mathcal{A}}}{\partial t} .
$$

The fluid-structure interface, namely $\partial \Omega_{f}(t) \cap \partial \Omega_{s}(t)$ is denoted by $\Sigma(t)$, and $\Gamma_{f}=$ $\partial \Omega_{f}(t) \backslash \Sigma(t)$ stands for the portion of the fluid boundary that is not shared with the boundary of the structure. The surface $\Gamma_{f}$ is assumed to be independent of $t$. The boundary $\partial \widehat{\Omega}_{s}$ of the reference configuration for the structure is divided into three disjoint parts $\widehat{\Gamma}_{D}, \widehat{\Gamma}_{N}$ and $\widehat{\Sigma}$, with $\Sigma(t)=\widehat{\mathcal{A}}_{t}(\widehat{\Sigma})$. We denote by $\boldsymbol{n}$ the outward unit normal on the fluid boundary in the current configuration, and by $\widehat{\boldsymbol{n}}_{s}$ the outward unit normal on the reference structure boundary. 


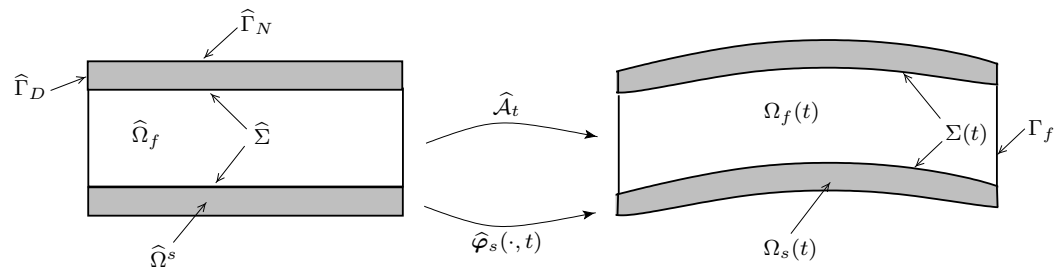

Figure 1. Parametrization of the domains $\Omega_{f}(t)$ and $\Omega_{s}(t)$

\subsection{The coupled problem}

We consider a homogeneous, Newtonian viscous, incompressible fluid with density $\rho_{f}$ and dynamic viscosity $\mu$. Its state is described by its Eulerian velocity $\boldsymbol{u}$ and pressure $p$. The constitutive law for the Cauchy stress tensor is given by the following expression:

$$
\boldsymbol{\sigma}(\boldsymbol{u}, p)=-p \boldsymbol{I}+2 \mu \boldsymbol{\epsilon}(\boldsymbol{u}),
$$

with $\boldsymbol{\epsilon}(\boldsymbol{u}) \stackrel{\text { def }}{=}\left[\nabla \boldsymbol{u}+(\nabla \boldsymbol{u})^{\mathrm{T}}\right] / 2$. In absence of body forces, these unknowns satisfy the incompressible Navier-Stokes equations in an ALE formulation:

$$
\left\{\begin{aligned}
\left.\rho_{f} \frac{\partial \boldsymbol{u}}{\partial t}\right|_{\widehat{\boldsymbol{x}}}+\rho_{f}(\boldsymbol{u}-\boldsymbol{w}) \cdot \boldsymbol{\nabla} \boldsymbol{u}-\operatorname{div}(2 \mu \boldsymbol{\epsilon}(\boldsymbol{u}))+\nabla p=0, & \text { in } \quad \Omega_{f}(t), \\
\operatorname{div} \boldsymbol{u}=0, & \text { in } \quad \Omega_{f}(t), \\
\boldsymbol{\sigma}(\boldsymbol{u}, p) \cdot \boldsymbol{n}=\boldsymbol{g}, & \text { on } \quad \Gamma_{f},
\end{aligned}\right.
$$

where $\left.\frac{\partial}{\partial t}\right|_{\widehat{\boldsymbol{x}}}$ stands for the ALE time derivative, $\boldsymbol{w} \stackrel{\text { def }}{=} \widehat{\boldsymbol{w}} \circ \widehat{\mathcal{A}}_{t}^{-1}$, and $\boldsymbol{g}$ a given density of surface force. We recall that $\Gamma_{f}$ is the complement of $\Sigma$ on $\partial \Omega_{f}$. Obviously, Dirichlet boundary conditions could also be considered, up to slight modifications in the presentation.

The structure is supposed to be hyperelastic under large displacements and deformations. Its density is denoted by $\rho_{s}$. Its state is described by its displacement $\widehat{\boldsymbol{d}}_{s}$ and its first Piola-Kirchoff stress tensor $\widehat{\boldsymbol{T}}$. The latter is related to $\widehat{\boldsymbol{d}}_{s}$ as the gradient of an internal stored energy function $W\left(\widehat{\boldsymbol{F}}_{s}\right)$. The choice of the internal stored energy will depend on the problem under consideration and will not change the setting of the fluid-structure problem. Assuming that the structure is clamped on $\Gamma_{D}$ and under no body and surface forces, these unknowns are driven by the following elastodynamic equations

$$
\left\{\begin{array}{rll}
\widehat{J}_{s} \rho_{s} \frac{\partial^{2} \widehat{\boldsymbol{d}}_{s}}{\partial t^{2}}-\operatorname{div} \widehat{\boldsymbol{x}} \widehat{\boldsymbol{T}}=\mathbf{0}, & \text { in } & \widehat{\Omega}_{s} \\
\widehat{\boldsymbol{d}}=\mathbf{0}, & \text { on } & \widehat{\Gamma}_{D} \\
\widehat{\boldsymbol{T}} \cdot \widehat{\boldsymbol{n}}_{s}=0, & \text { on } & \widehat{\Gamma}_{N}
\end{array}\right.
$$


The coupling between the solid and the fluid, namely equations [11] and [12], is realized through standard boundary conditions at the fluid-structure interface $\Sigma(t)$ that ensure the balance of the mechanical energy over the whole domain. This is achieved by imposing three interface conditions: face

- A geometrical condition enforcing the matching between $\varphi_{s}$ and $\widehat{\mathcal{A}}$ on the inter-

$$
\widehat{\boldsymbol{d}}_{f}=\widehat{\boldsymbol{d}}_{s}, \quad \text { on } \quad \widehat{\Sigma} .
$$

Inside $\widehat{\Omega}_{f}$, the fluid domain displacement $\widehat{\boldsymbol{d}}_{f}$ can be defined as an arbitrary (suitable) extension of $\widehat{\boldsymbol{d}}_{s}$ over the domain $\widehat{\Omega}_{f}$, namely,

$$
\widehat{\boldsymbol{d}}_{f}=\operatorname{Ext}\left(\widehat{\boldsymbol{d}}_{s \mid \widehat{\Sigma}}\right)
$$

(see Remark 3.1 below).

- A kinematic condition enforcing the continuity of the velocities at the interface

$$
\boldsymbol{u}=\frac{\partial \widehat{\boldsymbol{d}}_{s}}{\partial t} \circ \widehat{\mathcal{A}}_{t}^{-1}, \quad \text { on } \quad \Sigma(t)
$$

- And a kinetic condition imposing the stress continuity at the interface

$$
\widehat{\boldsymbol{T}} \widehat{\boldsymbol{n}}_{s}=-\widehat{J}_{f} \widehat{\boldsymbol{\sigma}(\boldsymbol{u}, p)} \widehat{\boldsymbol{F}}_{f}^{-T} \widehat{\boldsymbol{n}}, \quad \text { on } \quad \widehat{\Sigma} .
$$

To sum up, the fluid-structure system involving an incompressible viscous fluid and a hyperelastic structure is described in terms of the unknowns $\left(\boldsymbol{u}, p, \widehat{\boldsymbol{d}}_{f}, \widehat{\boldsymbol{d}}_{s}\right)$ satisfying the coupled problem [11]-[16].

REMARK. - In practice, we can choose as operator Ext a harmonic extension operator, by solving a Laplace equation

$$
\left\{\begin{array}{rll}
-\operatorname{div}\left(\kappa \nabla \widehat{\boldsymbol{d}}_{f}\right)=0, & \text { on } & \widehat{\Omega}_{f}, \\
\widehat{\boldsymbol{d}}_{f}=\widehat{\boldsymbol{d}}_{s}, & \text { on } & \widehat{\Sigma}, \\
\widehat{\boldsymbol{d}}_{f}=\mathbf{0}, & \text { on } & \widehat{\Gamma}_{f},
\end{array}\right.
$$

where $\kappa>0$ is a given "diffusion" coefficient, that may depend on $\widehat{\boldsymbol{d}}_{s}$. Alternative extensions can be found, for instance, in (Batina, 1990; Thomas et al., 1979).

REMARK. - The combination of [13] and [15] enforces $\boldsymbol{u}=\boldsymbol{w}$ on $\Sigma(t)$. This requirement is not strictly necessary but simplifies the construction of the ALE map. In general we could replace [14] by $\frac{\partial \widehat{\boldsymbol{d}}_{s}}{\partial t} \circ \widehat{\mathcal{A}}_{t}^{-1} \cdot \boldsymbol{n}=\boldsymbol{w} \cdot \boldsymbol{n}$ on $\Sigma(t)$.

REMARK. - For simplicity, we have only prescribed Neumann boundary conditions in [11]. In practice we may use Dirichlet conditions on some part of the boundary. 


\subsection{Weak formulation}

Problem [11]-[16] can be reformulated in a weak variational form using appropriate test functions, performing integrations by parts and taking into account the boundary and interface conditions.

In what follows, we will make explicit the dependence of $\Omega_{f}(t)$ and $\Sigma(t)$ on $\widehat{\boldsymbol{d}}_{f}$ by introducing the notation

$$
\Omega_{f}\left(\widehat{\boldsymbol{d}}_{f}\right) \stackrel{\text { def }}{=} \Omega_{f}(t), \quad \Sigma\left(\widehat{\boldsymbol{d}}_{f}\right) \stackrel{\text { def }}{=} \Sigma(t) .
$$

The global weak formulation of problem [11]-[16] (derived in Appendix A) reads: Find $\widehat{\boldsymbol{u}}: \widehat{\Omega}_{f} \times \mathbb{R}^{+} \rightarrow \mathbb{R}^{3}, \widehat{p}: \widehat{\Omega}_{f} \times \mathbb{R}^{+} \rightarrow \mathbb{R}, \widehat{\boldsymbol{d}}_{f}: \widehat{\Omega}_{f} \times \mathbb{R}^{+} \rightarrow \mathbb{R}^{3}, \widehat{\boldsymbol{d}}_{s}: \widehat{\Omega}_{s} \times \mathbb{R}^{+} \rightarrow \mathbb{R}^{3}$ and $\gamma: \widehat{\Sigma} \times \mathbb{R}^{+} \rightarrow \mathbb{R}^{3}$ such that

$$
\begin{aligned}
& \frac{\mathrm{d}}{\mathrm{dt}} \int_{\Omega_{f}\left(\widehat{\boldsymbol{d}_{f}}\right)} \rho_{f} \boldsymbol{u} \cdot \boldsymbol{v}_{f} \mathrm{~d} \boldsymbol{x}+\int_{\Omega_{f}\left(\widehat{\boldsymbol{d}_{f}}\right)} \operatorname{div}\left[\rho_{f} \boldsymbol{u} \otimes\left(\boldsymbol{u}-\boldsymbol{w}\left(\widehat{\boldsymbol{d}_{f}}\right)\right)\right] \cdot \boldsymbol{v}_{f} \mathrm{~d} \boldsymbol{x} \\
& +\int_{\Omega_{f}\left(\widehat{\boldsymbol{d}_{f}}\right)} \boldsymbol{\sigma}(\boldsymbol{u}, p): \nabla \boldsymbol{v}_{f} \mathrm{~d} \boldsymbol{x}-\int_{\Gamma_{\mathrm{in}-\mathrm{out}}} \boldsymbol{g} \cdot \boldsymbol{v}_{f} \mathrm{~d} \boldsymbol{a}-\int_{\Omega_{f}\left(\widehat{\boldsymbol{d}_{f}}\right)} q \operatorname{div} \boldsymbol{u} \mathrm{d} \boldsymbol{x} \\
& +\int_{\widehat{\Omega_{s}}} \rho_{0} \frac{\partial^{2} \widehat{\boldsymbol{d}_{s}}}{\partial t^{2}} \cdot \widehat{\boldsymbol{v}_{s}} \mathrm{~d} \hat{\boldsymbol{x}}+\int_{\widehat{\Omega_{s}}} \frac{\partial W}{\partial F}\left(\mathrm{I}+\nabla \widehat{\boldsymbol{d}_{s}}\right): \nabla \widehat{\boldsymbol{v}_{s}} \mathrm{~d} \hat{\boldsymbol{x}} \\
& +\int_{\widehat{\Omega_{f}}}\left(\widehat{\boldsymbol{d}_{f}}-\operatorname{Ext}(\gamma)\right) \cdot \widehat{\boldsymbol{\tau}} \mathrm{d} \hat{\boldsymbol{x}}+\int_{\widehat{\Sigma}}\left(\widehat{\boldsymbol{d}_{s}}-\gamma\right) \cdot \widehat{\boldsymbol{\zeta}} \mathrm{d} \hat{\boldsymbol{a}} \\
& +\int_{\widehat{\Sigma}}\left(\widehat{\boldsymbol{u}}-\widehat{\boldsymbol{w}}\left(\widehat{\boldsymbol{d}_{f}}\right)\right) \cdot \widehat{\boldsymbol{\xi}} \mathrm{d} \hat{\boldsymbol{a}}=0
\end{aligned}
$$

with $\boldsymbol{u}(\cdot, t)=\widehat{\boldsymbol{u}}(\cdot, t) \circ \widehat{\mathcal{A}}_{t}^{-1}, p(\cdot, t)=\widehat{p}(\cdot, t) \circ \widehat{\mathcal{A}}_{t}^{-1}$, and for all $\left(\widehat{\boldsymbol{v}_{f}}, \widehat{q}\right) \in\left[H^{1}\left(\widehat{\Omega}_{f}\right)\right]^{3} \times$ $L^{2}\left(\widehat{\Omega}_{f}\right), \widehat{\boldsymbol{v}}_{s} \in\left[H_{\widehat{\Gamma}_{D}}^{1}\left(\widehat{\Omega}_{s}\right)\right]^{3}$ with $\widehat{\boldsymbol{v}_{f}}=\widehat{\boldsymbol{v}_{s}}$ on $\widehat{\Sigma}, \widehat{\boldsymbol{\tau}} \in\left[L^{2}\left(\widehat{\Omega}_{f}\right)\right]^{3}, \widehat{\boldsymbol{\zeta}} \in\left[L^{2}(\widehat{\Sigma})\right]^{3}$ and $\widehat{\boldsymbol{\xi}} \in\left[L^{2}(\widehat{\Sigma})\right]^{3}$, where $\left[H_{\widehat{\Gamma}_{D}}^{1}\left(\widehat{\Omega}_{s}\right)\right]^{3}$ denotes the subspace of functions of $\left[H^{1}\left(\widehat{\Omega}_{s}\right)\right]^{3}$ vanishing on $\widehat{\Gamma}_{D}$.

\section{Constitutive laws for artery walls}

\subsection{Three dimensional constitutive laws}

In an extensive survey article (Holzapfel et al., 2000), Holzapfel et al. have analyzed and compared existing constitutive models for arterial walls. They have also introduced a new framework to take into account anisotropy and various mechanical effects such as inflation and torsion. Their model is based on a thick-walled nonlinearly elastic tube consisting of two layers. Another model has been introduced by van Oijen in his $\mathrm{PhD}$ thesis (Oijen, 2003). More microscopically based, it uses the mixing 
theory to take into account the fibers in the layers. Even more precise at the microscopic level, Caillerie et al. have introduced a nonlinear homogenization approach to fiber-reinforcement in soft tissues (Caillerie et al., 2003).

These three models have two common features: they are three-dimensional and anisotropic. Previous approaches, such as the Fung model in (Fung et al., 1979), are based on geometrical simplifications, such as membrane, and more generally on thin shell. However, as pointed out in (Holzapfel et al., 2000), such simplifications are not suitable for the analysis of the through-thickness stress distribution in an artery or for the treatment of shearing deformations. In addition, the combination of inflation and torsion effects cannot be reproduced by such simplified models. This may explain why three-dimensional constitutive laws are needed to correctly handle the passive mechanical behavior of artery walls.

From a physiological point of view, the arterial wall is made of three layers (the intima, the media and the adventitia). For a healthy artery, only the media and the adventitia have a significant mechanical role. In addition, their mechanical behavior is highly anisotropic due to the presence of fibers (collagenous components). In (Holzapfel et al., 2000), Holzapfel et al. propose a model based on two layers modeling the media and the adventitia. For both layers, the material is supposed to be threedimensional, thin, hyperelastic, in finite deformation, incompressible, anisotropic (in the fiber directions) and pre-stressed.

The elastic assumption is well satisfied in some vessels, as the aorta, the iliac and carotid arteries. For other arteries, including the femoral, celiac and cerebral arteries, viscoelastic models are needed.

As a consequence of the above assumptions, the free energy of a layer can be written as

$$
W\left(\widehat{\boldsymbol{F}}_{s}\right)=\Psi_{\text {iso }}\left(I_{1}, I_{2}, J\right)+\Psi_{\mathrm{fib}}\left(I_{4}, I_{5}\right),
$$

where $\widehat{\boldsymbol{F}}_{s}$ is the deformation gradient, $I_{1}, I_{2}$ and $J$ its three principal invariants and $I_{4}$ and $I_{5}$ its pseudo-invariants related to the reinforcement direction. The first part of the energy $\Psi_{\text {iso }}$ is isotropic, typically a neo-Hookean, Mooney-Rivlin or CiarletGeymonat type of energy. The second part $\Psi_{\text {fib }}$ is anisotropic and involves an exponential term in order to reproduce the strong stiffening effect of each layer at high pressure.

From a computational point of view, the above combination of mechanical properties gives rise to two major difficulties: the treatment of incompressibility in finite deformation and the treatment of bad aspect ratios for thin three-dimensional structures. Both phenomena lead to locking problems ((Chapelle et al., 2003a),(Chapelle et al., 2003c)) if not correctly treated. Incompressibility issues are classically dealt with using a mixed finite element method, whereas locking phenomena in thin three-dimensional structures are treated using re-interpolation techniques (Chapelle et al., 2003b; Chapelle et al., 2003c; Chapelle et al., 2004) as presented in the following subsection. 


\section{2. $3 D$ shell elements}

A general structural model of the blood flow with complex and realistic geometries has to be three-dimensional and handle large displacements.

Since the walls of the blood vessels are thin, it is convenient to use shell elements; they accurately describe its geometry. All finite elements adopted in our simulations are general shell elements. Previously, Gerbeau et al. have used the MITC4 elements (Gerbeau et al., 2003; Gerbeau et al., 2005). This element is a 4 node quadrangle ( 5 degrees of freedom per node) with a 3D constitutive law for which the transversal stress is null and a kinematic constraint is needed to make the model compatible with a Reissner-Mindlin shell model. This restricts the choice of the energy. The MITC technique is used to avoid locking (Chapelle et al., 2003a).

We consider here 3D shell elements (Chapelle et al., 2003b; Chapelle et al., 2003c; Chapelle et al., 2004). Geometrically, these elements appear as standard 27 nodes three-dimensional elements. Thus it is very easy to couple them to other threedimensional formulations through the nodes on the faces, whereas the quadratic approximation in the shell's thickness allows one to deal with standard 3D energies, such as generalized Hook or any hyperelastic stored energy, defined by using the CauchyGreen tensor's invariants, such as in [19]. Yet, since the hexahedra are very elongated in the plane orthogonal to the thin direction, standard $3 \mathrm{D} \mathbb{Q}_{2}$ shape functions with a standard integration rule would exhibit locking as the meshsize vanishes. The element considered here, called MI3D, uses standard 3D $\mathbb{Q}_{2}$ shape functions but a specific integration rule. The idea introduced and analyzed in (Chapelle et al., 2003b; Chapelle et al., 2003c; Chapelle et al., 2004) consists in using the reinterpolation technique specifically developed for shell elements in (Chapelle et al., 2003a). This produces a locking-free FE method. This strategy is in the same spirit as the use of methods designed for incompressible materials (mixed finite elements, or subintegration) to prevent locking for quasi-incompressible materials.

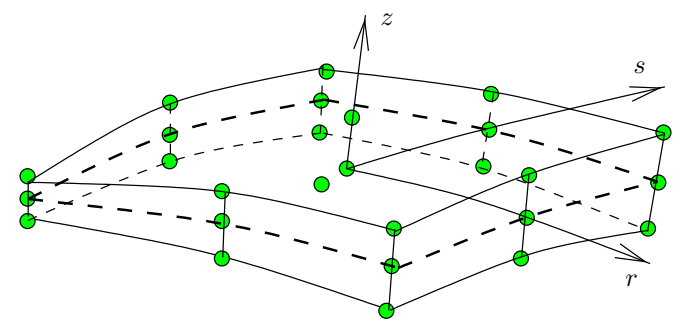

Figure 2. $3 D$ shell element

In order to be able to apply MITC techniques to stabilize the formulation, it is necessary to compute the first and second derivatives of the stored energy with respect 
to the Green-Lagrange tensor, defined hereafter, in local coordinates $(r, s, z)$, as it is usually done for shell elements (see Figure 2):

$$
e_{i j}(\vec{U}) \stackrel{\text { def }}{=} \frac{1}{2}\left(\vec{g}_{i} \cdot \vec{U}_{, j}+\vec{g}_{j} \cdot \vec{U}_{, i}+\vec{U}_{, i} \cdot \vec{U}_{, j}\right),
$$

where $\vec{g}_{i}$ is a covariant basis.

The first and second order infinitesimal variations are given by

$$
\begin{aligned}
\delta e_{i j} & =\frac{1}{2}\left(\vec{g}_{i} \cdot \delta \vec{U}_{, j}+\vec{g}_{j} \cdot \delta \vec{U}_{, i}+\vec{U}_{, i} \cdot \delta \vec{U}_{, j}+\vec{U}_{, j} \cdot \delta \vec{U}_{, i}\right), \\
d \delta e_{i j} & =\frac{1}{2}\left(d \vec{U}_{, i} \cdot \delta \vec{U}_{, j}+d \vec{U}_{, j} \cdot \delta \vec{U}_{, i}\right) .
\end{aligned}
$$

When dealing with elastodynamics, at each time step of, say, a Newmark timediscretization, a nonlinear problem has to be solved. The bilinear form appearing in this algorithm is the following:

$$
A=A^{L}+A^{N L},
$$

with

$$
\begin{gathered}
A^{L}(d \vec{U}, \delta \vec{U}) \stackrel{\text { def }}{=} \int_{\Omega} \frac{\partial^{2} W}{\partial e_{i j} \partial e_{k l}} d e_{k l} \delta e_{i j} d V, \\
A^{N L}(d \vec{U}, \delta \vec{U}) \stackrel{\text { def }}{=} \int_{\Omega} \frac{\partial W}{\partial e_{i j}} d \delta e_{i j} d V,
\end{gathered}
$$

and the corresponding nonlinear right-hand side

$$
F^{N L}(\delta \vec{U}) \stackrel{\text { def }}{=} \int_{\Omega} \frac{\partial W}{\partial e_{i j}} \delta e_{i j} d V .
$$

In practice, the values of the deformation are not directly computed by [20], but are re-interpolated at the tying points defined by MITC methods. The first and second order infinitesimal variations in [21]-[23] have to be re-interpolated using the same rules in order to obtain a consistent tangent problem.

Both the MITC4 and the MI3D elements can be employed in actual computations. The MITC4 with 4 nodes and 5 degrees of freedom per node has 20 degrees of freedom per element, the MI3D with 27 nodes and 3 degrees of freedom per node has 81 degrees of freedom per element. The MI3D is indeed more expensive than the MITC4, but it is also more practical for realistic models of the arteries, as recalled at the beginning of this section.

In Section 7, we present some numerical tests using (from one to three layers of) MI3D elements with a neo-Hookean constitutive law in finite deformation, thus tackling the two major numerical difficulties for the implementation of the thick-walled nonlinearly elastic bilayer constitutive laws introduced in (Holzapfel et al., 2000). This example provides us with a case of interest for which the numerical method developed in Section 6 may be competitive. 


\section{Semi-discretized weak formulation}

In this section, the weak coupled formulation [18] is semi-discretized in time using an implicit coupling-scheme. The resulting nonlinear problem will be turned into an abstract form. This will allow us to introduce in the next section general nonlinear iterative solution methods.

\subsection{Implicit coupling scheme}

We use an implicit Euler scheme for the ALE Navier-Stokes equations, with a semi-implicit treatment of the nonlinear convective term. Furthermore we use a midpoint rule for the structural equation. Thus, given a time step $\delta t>0$, for $n=0,1, \ldots$, the time semi-discretized coupled problem writes: Given $\left(\widehat{\boldsymbol{u}}^{n}, \widehat{p}^{n}, \widehat{\boldsymbol{d}}_{f}^{n}, \widehat{\boldsymbol{d}}_{s}^{n}, \gamma^{n}\right)$, find

$$
\begin{array}{r}
\left(\widehat{\boldsymbol{u}}^{n+1}, \widehat{p}^{n+1}, \widehat{\boldsymbol{d}}^{n+1}, \widehat{\boldsymbol{d}}^{n+1}, \boldsymbol{\gamma}^{n+1}\right) \in\left[H^{1}\left(\widehat{\Omega}_{f}\right)\right]^{3} \times L^{2}\left(\widehat{\Omega}_{f}\right) \times\left[H^{1}\left(\widehat{\Omega}_{f}\right)\right]^{3} \\
\times\left[H^{1}\left(\widehat{\Omega}_{s}\right)\right]^{3} \times\left[H^{\frac{1}{2}}(\widehat{\Sigma})\right]^{3}
\end{array}
$$

such that

$$
\begin{aligned}
& \frac{1}{\delta t} \int_{\Omega_{f}\left(\widehat{\boldsymbol{d}}_{f}^{n+1}\right)} \rho_{f} \boldsymbol{u}^{n+1} \cdot \boldsymbol{v}_{f} \mathrm{~d} \boldsymbol{x}-\frac{1}{\delta t} \int_{\Omega_{f}\left(\widehat{\boldsymbol{d}}_{f}^{n}\right)} \rho_{f} \boldsymbol{u}^{n} \cdot \boldsymbol{v}_{f} \mathrm{~d} \boldsymbol{x} \\
& +\int_{\Omega_{f}\left(\widehat{\boldsymbol{d}}_{f}^{n+1}\right)} \boldsymbol{\sigma}\left(\boldsymbol{u}^{n+1}, p^{n+1}\right): \nabla \boldsymbol{v}_{f} \mathrm{~d} \boldsymbol{x} \\
& +\int_{\Omega_{f}\left(\widehat{\boldsymbol{d}}_{f}^{n+1}\right)} \operatorname{div}\left[\rho_{f} \boldsymbol{u}^{n+1} \otimes\left(\boldsymbol{u}^{n}-\boldsymbol{w}\left(\widehat{\boldsymbol{d}}_{f}^{n+1}\right)\right)\right] \cdot \boldsymbol{v}_{f} \mathrm{~d} \boldsymbol{x} \\
& -\int_{\Gamma_{\mathrm{in}-\mathrm{out}}} \boldsymbol{g}^{n+1} \cdot \boldsymbol{v}_{f} \mathrm{~d} \boldsymbol{a}-\int_{\Omega_{f}\left(\widehat{\boldsymbol{d}}_{f}^{n+1}\right)} q \operatorname{div} \boldsymbol{u}^{n+1} \mathrm{~d} \boldsymbol{x} \\
& +\int_{\widehat{\Omega}_{f}}\left(\widehat{\boldsymbol{d}}_{f}^{n+1}-\operatorname{Ext}\left(\gamma^{n+1}\right)\right) \cdot \widehat{\boldsymbol{\tau}} \mathrm{d} \hat{\boldsymbol{x}} \\
& +\int_{\widehat{\Sigma}}\left(\widehat{\boldsymbol{u}}^{n+1}-\widehat{\boldsymbol{w}}\left(\widehat{\boldsymbol{d}}_{f}^{n+1}\right)\right) \cdot \widehat{\boldsymbol{\xi}} \mathrm{d} \hat{\boldsymbol{a}}+\frac{2}{\delta t^{2}} \int_{\widehat{\Omega}_{s}} \rho_{0} \widehat{\boldsymbol{d}}_{s}^{n+1} \cdot \widehat{\boldsymbol{v}}_{s} \mathrm{~d} \hat{\boldsymbol{x}} \\
& -\frac{2}{\delta t^{2}} \int_{\widehat{\Omega}_{s}} \rho_{0}\left(\widehat{\boldsymbol{d}}_{s}^{n}+\delta t \dot{\hat{\boldsymbol{d}}_{s}^{n}}\right) \cdot \widehat{\boldsymbol{v}}_{s} \mathrm{~d} \hat{\boldsymbol{x}} \\
& +\int_{\widehat{\Omega}_{s}} \frac{\partial W}{\partial F}\left(I+\frac{1}{2} \nabla\left(\widehat{\boldsymbol{d}}_{s}^{n}+\widehat{\boldsymbol{d}}_{s}^{n+1}\right)\right): \nabla \widehat{\boldsymbol{v}}_{s} \mathrm{~d} \hat{\boldsymbol{x}} \\
& +\int_{\widehat{\Sigma}}\left(\widehat{\boldsymbol{d}}_{s}^{n+1}-\gamma^{n+1}\right) \cdot \widehat{\boldsymbol{\zeta}} \mathrm{d} \hat{\boldsymbol{a}}=0,
\end{aligned}
$$


for all $\left(\widehat{\boldsymbol{v}_{f}}, \widehat{q}, \widehat{\boldsymbol{\xi}}, \widehat{\boldsymbol{\tau}}, \widehat{\boldsymbol{\zeta}}, \widehat{\boldsymbol{v}}_{s}\right) \in\left[H^{1}\left(\widehat{\Omega}_{f}\right)\right]^{3} \times L^{2}\left(\widehat{\Omega}_{f}\right) \times\left[L^{2}(\widehat{\Sigma})\right]^{3} \times\left[L^{2}\left(\widehat{\Omega}_{f}\right)\right]^{3} \times\left[L^{2}(\widehat{\Sigma})\right]^{3} \times$ $\left[H_{\widehat{\Gamma}_{D}}^{1}\left(\widehat{\Omega}_{s}\right)\right]^{3}$ such that $\widehat{\boldsymbol{v}_{f}}=\widehat{\boldsymbol{v}_{s}}$ on $\widehat{\Sigma}$, and with $\boldsymbol{u}^{n}=\widehat{\boldsymbol{u}}^{n} \circ\left(\boldsymbol{I}+{\widehat{\boldsymbol{d}_{f}}}^{n}\right)^{-1}$ (analogously for $\left.p^{n}\right)$ and $\widehat{\boldsymbol{d}}_{s}^{\dot{n}+1}=\frac{2}{\delta t}\left(\widehat{\boldsymbol{d}}_{s}^{n+1}-\widehat{\boldsymbol{d}}_{s}^{n}\right)-\dot{\hat{\boldsymbol{d}_{s}^{n}}}$.

\subsection{Abstract formulations}

Problem [24] can be rewritten in a more compact form in terms of the fluid, solid and interface state operators.

Based on the discrete weak formulation [24] we introduce the fluid operator

$$
\begin{aligned}
\mathcal{F}:\left[H^{1}\left(\widehat{\Omega}_{f}\right)\right]^{3} \times & L^{2}\left(\widehat{\Omega}_{f}\right) \times\left[H^{1}\left(\widehat{\Omega}_{f}\right)\right]^{3} \times\left[H^{\frac{1}{2}}(\widehat{\Sigma})\right]^{3} \\
& \longrightarrow\left(\left[H_{\widehat{\Sigma}}^{1}\left(\widehat{\Omega}_{f}\right)\right]^{3} \times L^{2}\left(\widehat{\Omega}_{f}\right) \times\left[L^{2}(\widehat{\Sigma})\right]^{3} \times\left[L^{2}\left(\widehat{\Omega}_{f}\right)\right]^{3}\right)^{\prime},
\end{aligned}
$$

defined by

$$
\begin{aligned}
& \left\langle\mathcal{F}\left(\widehat{\boldsymbol{u}}, \widehat{p}, \widehat{\boldsymbol{d}}_{f}, \gamma\right),\left(\widehat{\boldsymbol{v}_{f}}, \widehat{\mathrm{q}}, \widehat{\boldsymbol{\xi}}, \widehat{\boldsymbol{\tau}}\right)\right\rangle=\frac{1}{\delta t} \int_{\Omega_{f}\left(\widehat{\boldsymbol{d}}_{f}\right)} \rho_{f} \boldsymbol{u} \cdot \boldsymbol{v}_{f} \mathrm{~d} \boldsymbol{x} \\
& \quad-\frac{1}{\delta t} \int_{\Omega_{f}\left(\widehat{\boldsymbol{d}}_{f}^{n}\right)} \rho_{f} \boldsymbol{u}^{n} \cdot \boldsymbol{v}_{f} \mathrm{~d} \boldsymbol{x} \\
& \quad+\int_{\Omega_{f}\left(\widehat{\boldsymbol{d}}_{f}\right)} \operatorname{div}\left[\rho_{f} \boldsymbol{u} \otimes\left(\boldsymbol{u}^{n}-\boldsymbol{w}\left(\widehat{\boldsymbol{d}}_{f}\right)\right)\right] \cdot \boldsymbol{v}_{f} \mathrm{~d} \boldsymbol{x} \\
& \quad+\int_{\Omega_{F}\left(\widehat{\boldsymbol{d}}_{f}\right)} \boldsymbol{\sigma}(\boldsymbol{u}, p): \nabla \boldsymbol{v}_{f} \mathrm{~d} \boldsymbol{x}-\int_{\Gamma_{\mathrm{in}-\mathrm{out}}\left(\widehat{\boldsymbol{d}}_{f}\right)} \boldsymbol{g}^{n+1} \cdot \boldsymbol{v}_{f} \mathrm{~d} \boldsymbol{a} \\
& \quad-\int_{\Omega_{f}\left(\widehat{\boldsymbol{d}}_{f}\right)} q \operatorname{div} \boldsymbol{u} \mathrm{d} \boldsymbol{x}+\int_{\widehat{\Sigma}}\left(\widehat{\boldsymbol{u}}-\widehat{\boldsymbol{w}}\left(\widehat{\boldsymbol{d}}_{f}\right)\right) \cdot \widehat{\boldsymbol{\xi}} \mathrm{d} \hat{\boldsymbol{a}} \\
& \quad+\int_{\widehat{\Omega}_{f}}\left(\widehat{\boldsymbol{d}}_{f}-\operatorname{Ext}(\gamma)\right) \cdot \widehat{\boldsymbol{\tau}} \mathrm{d} \hat{\boldsymbol{x}},
\end{aligned}
$$

for all $\left(\widehat{\boldsymbol{v}_{f}}, \widehat{q}, \widehat{\boldsymbol{\xi}}, \widehat{\boldsymbol{\tau}}\right) \in\left[H^{1}\left(\widehat{\Omega}_{f}\right)\right]^{3} \times L^{2}\left(\widehat{\Omega}_{f}\right) \times\left[L^{2}(\widehat{\Sigma})\right]^{3} \times\left[L^{2}\left(\widehat{\Omega}_{f}\right)\right]^{3}$.

Analogously, from [24], the solid operator

$$
\mathcal{S}:\left[H^{1}\left(\widehat{\Omega}_{s}\right)\right]^{3} \times\left[H^{\frac{1}{2}}(\widehat{\Sigma})\right]^{3} \longrightarrow\left(\left[H_{\Gamma_{D} \cup \widehat{\Sigma}}^{1}\left(\widehat{\Omega}_{s}\right)\right]^{3} \times\left[L^{2}(\widehat{\Sigma})\right]^{3}\right)^{\prime},
$$

is given by

$$
\begin{array}{r}
\left\langle\mathcal{S}\left(\widehat{\boldsymbol{d}}_{s}, \boldsymbol{\gamma}\right),\left(\widehat{\boldsymbol{v}_{s}}, \widehat{\boldsymbol{\zeta}}\right)\right\rangle=\frac{2}{\delta t^{2}} \int_{\widehat{\Omega}_{s}} \rho_{0} \widehat{\boldsymbol{d}}_{s} \cdot \boldsymbol{v}_{s} \mathrm{~d} \hat{\boldsymbol{x}}-\frac{2}{\delta t^{2}} \int_{\widehat{\Omega}_{s}} \rho_{0}\left(\widehat{\boldsymbol{d}}_{s}^{n}+\delta t \dot{\hat{\boldsymbol{d}}_{s}^{n}}\right) \cdot \boldsymbol{v}_{s} \mathrm{~d} \hat{\boldsymbol{x}} \\
+\int_{\widehat{\Omega}_{s}} \frac{\partial W}{\partial F}\left(I+\frac{1}{2} \nabla\left(\widehat{\boldsymbol{d}}_{s}^{n}+\widehat{\boldsymbol{d}}_{s}\right)\right): \nabla \widehat{\boldsymbol{v}}_{s} \mathrm{~d} \hat{\boldsymbol{x}}+\int_{\widehat{\Sigma}}\left(\widehat{\boldsymbol{d}}_{s}-\gamma\right) \cdot \widehat{\boldsymbol{\zeta}} \mathrm{d} \hat{\boldsymbol{a}},
\end{array}
$$


for all $\left(\widehat{\boldsymbol{v}_{s}}, \widehat{\boldsymbol{\zeta}}\right) \in\left[H_{\Gamma_{D}}^{1}\left(\widehat{\Omega}_{s}\right)\right]^{3} \times\left[L^{2}(\widehat{\Sigma})\right]^{3}$

Finally, let

$$
\mathcal{L}_{f}:\left[H^{\frac{1}{2}}(\widehat{\Sigma})\right]^{3} \rightarrow\left[H_{\Gamma_{\text {in }- \text { out }}}^{1}\left(\widehat{\Omega}_{f}\right)\right]^{3}
$$

and

$$
\mathcal{L}_{s}:\left[H^{\frac{1}{2}}(\widehat{\Sigma})\right]^{3} \rightarrow\left[H_{\partial \widehat{\Omega}_{s} \backslash \widehat{\Sigma}}^{1}\left(\widehat{\Omega}_{s}\right)\right]^{3}
$$

be two given continuous linear lift operators. The interface operator

$$
\mathcal{I}:\left[H^{1}\left(\widehat{\Omega}_{f}\right)\right]^{3} \times L^{2}\left(\widehat{\Omega}_{f}\right) \times\left[H^{1}\left(\widehat{\Omega}_{f}\right)\right]^{3} \times\left[H^{1}\left(\widehat{\Omega}_{s}\right)\right]^{3} \longrightarrow\left[H^{-\frac{1}{2}}(\widehat{\Sigma})\right]^{3},
$$

is then defined by

$$
\begin{aligned}
\left\langle\mathcal{I}\left(\widehat{\boldsymbol{u}}, \widehat{p}, \widehat{\boldsymbol{d}}_{f}, \widehat{\boldsymbol{d}}_{s}\right), \boldsymbol{\mu}\right\rangle=\left\langle\mathcal{F}\left(\widehat{\boldsymbol{u}}, \widehat{p}, \widehat{\boldsymbol{d}}_{f}, \gamma\right),\right. & \left.\left(\mathcal{L}_{f} \boldsymbol{\mu}, 0, \mathbf{0}, \mathbf{0}\right)\right\rangle \\
& +\left\langle\mathcal{S}\left(\widehat{\boldsymbol{d}}_{s}, \boldsymbol{\gamma}\right),\left(\mathcal{L}_{s} \boldsymbol{\mu}, \mathbf{0}\right)\right\rangle,
\end{aligned}
$$

for all $\boldsymbol{\mu} \in\left[H^{\frac{1}{2}}(\widehat{\Sigma})\right]^{3}$.

REMARK. - The interface operator does not depend on $\gamma$ since, due to the choice of the test functions, the terms involving $\gamma$ vanish in the right-hand side of [27].

According to the above definitions, problem [24] is equivalent to

$$
\text { Formulation (I): } \quad\left\{\begin{aligned}
\mathcal{F}\left(\widehat{\boldsymbol{u}}^{n+1}, \widehat{p}^{n+1}, \widehat{\boldsymbol{d}}_{f}^{n+1}, \boldsymbol{\gamma}^{n+1}\right) & =0, \\
\mathcal{S}\left(\widehat{\boldsymbol{d}}_{s}^{n+1}, \boldsymbol{\gamma}^{n+1}\right) & =0, \\
\mathcal{I}\left(\widehat{\boldsymbol{u}}^{n+1}, \widehat{p}^{n+1}, \widehat{\boldsymbol{d}}_{f}^{n+1}, \widehat{\boldsymbol{d}}_{s}^{n+1}\right) & =0 .
\end{aligned}\right.
$$

\subsection{Steklov-Poincaré operators}

In order to describe partitioned methods for the numerical solution of [24], we now introduce the nonlinear fluid and solid Steklov-Poincaré operators.

The nonlinear fluid Steklov-Poincaré operator

$$
S_{f}:\left[H^{\frac{1}{2}}(\widehat{\Sigma})\right]^{3} \longrightarrow\left[H^{-\frac{1}{2}}(\widehat{\Sigma})\right]^{3},
$$

is defined by

$$
\left\langle S_{f}(\gamma), \boldsymbol{\mu}\right\rangle=\left\langle\mathcal{I}\left(\widehat{\boldsymbol{u}}(\boldsymbol{\gamma}), \widehat{p}(\boldsymbol{\gamma}), \widehat{\boldsymbol{d}}_{f}(\gamma), \mathbf{0}\right), \boldsymbol{\mu}\right\rangle,
$$

for all $\gamma, \boldsymbol{\mu} \in\left[H^{\frac{1}{2}}(\hat{\Sigma})\right]^{3}$, where $\left(\widehat{\boldsymbol{u}}(\gamma), \widehat{p}(\gamma), \widehat{\boldsymbol{d}}_{f}(\gamma)\right)$ is the solution of the Dirichlet fluid problem:

$$
\mathcal{F}\left(\widehat{\boldsymbol{u}}(\gamma), \widehat{p}(\gamma), \widehat{\boldsymbol{d}_{f}}(\gamma), \gamma\right)=0
$$


In an analogous way, we introduce the nonlinear solid Steklov-Poincaré operator

$$
S_{s}:\left[H^{\frac{1}{2}}(\hat{\Sigma})\right]^{3} \longrightarrow\left[H^{-\frac{1}{2}}(\hat{\Sigma})\right]^{3},
$$

given by

$$
\left\langle S_{s}(\gamma), \boldsymbol{\mu}\right\rangle=\left\langle\mathcal{I}\left(\mathbf{0}, 0, \mathbf{0}, \widehat{\boldsymbol{d}}_{s}(\gamma)\right), \boldsymbol{\mu}\right\rangle
$$

for all $\gamma, \boldsymbol{\mu} \in\left[H^{\frac{1}{2}}(\hat{\Sigma})\right]^{3}$ and where $\widehat{\boldsymbol{d}_{s}}(\gamma)$ is the solution of the Dirichlet solid problem:

$$
\mathcal{S}\left(\widehat{\boldsymbol{d}}_{s}(\gamma), \gamma\right)=0
$$

From the above definitions, it follows that problem [24] (or [28]) is equivalent to

$$
\text { Formulation (II): } \quad S_{f}(\gamma)+S_{s}(\gamma)=0 .
$$

The composition of [29] with the inverse operators $S_{s}^{-1}$ gives rise to the Dirichlet-toNeumann formulation, namely

$$
\text { Formulation (III): } \quad S_{s}^{-1}\left(-S_{f}(\gamma)\right)-\gamma=0 .
$$

We could also consider the Neumann-to-Dirichlet formulation

$$
S_{f}^{-1}\left(-S_{s}(\gamma)\right)-\gamma=0
$$

by composing [29] with $S_{f}^{-1}$. Nevertheless it is rarely used in practice and it is known to lead to poor algorithms in some cases, as pointed out in (Causin et al., 2005).

\section{A partitioned Newton method}

In what follows, we skip the upper script $n$ since the time step is fixed. The method presented here consists in solving [28] by a Newton method: given an initial guess $\left(\widehat{\boldsymbol{u}}_{0}, \widehat{p}_{0}, \widehat{\boldsymbol{d}}_{f}^{0}, \widehat{\boldsymbol{d}}_{s}^{0}, \gamma_{0}\right)$, the algorithm reads

1) Evaluate the nonlinear residual of problem [28].

2) Solve the tangent problem (see [31] below) by a domain decomposition method.

3) Update solution:

$$
\left(\widehat{\boldsymbol{u}}, \widehat{p}, \widehat{\boldsymbol{d}}_{f}, \widehat{\boldsymbol{d}}_{s}, \gamma\right) \leftarrow\left(\widehat{\boldsymbol{u}}, \widehat{p}, \widehat{\boldsymbol{d}}_{f}, \widehat{\boldsymbol{d}}_{s}, \gamma\right)+\left(\delta \widehat{\boldsymbol{u}}, \delta \widehat{p}, \delta \widehat{\boldsymbol{d}}_{f}, \delta \widehat{\boldsymbol{d}}_{s}, \delta \gamma\right)
$$

4) repeat until convergence.

Compared to the known fluid-structure algorithms presented in Section 2.2, this partitioned Newton method amounts to switching the domain decomposition and the linearization in the resolution of the coupled problem. We provide the tangent problem in the following sections, as well as details for the domain decomposition resolution. 


\subsection{Abstract formulation}

In this section, we present an abstract formulation making use of the derivative of the fluid, structure and interface operators of Section 5.2 with respect to their arguments. The derivation uses shape derivative calculus for the differentiation of integral terms with respect to their supports. The precise formulae are given in Appendix B, and we refer the reader to (Fernández et al., 2005) where this issue is addressed (see also (Dettmer et al., 2006; Bazilevs et al., 2008; van der Zee et al., 2010)).

We thus introduce a linearized fluid operator at state $\left(\widehat{\boldsymbol{u}}, \widehat{p}, \widehat{\boldsymbol{d}}_{f}, \gamma\right) \in\left[H^{1}\left(\widehat{\Omega}_{f}\right)\right]^{3} \times$ $L^{2}\left(\widehat{\Omega}_{f}\right) \times\left[H^{1}\left(\widehat{\Omega}_{f}\right)\right]^{3} \times\left[H^{\frac{1}{2}}(\widehat{\Sigma})\right]^{3}$, denoted by

$$
\begin{aligned}
\operatorname{DF}\left(\widehat{\boldsymbol{u}}, \widehat{p}, \widehat{\boldsymbol{d}}_{f}, \gamma\right):\left[H^{1}\left(\widehat{\Omega}_{f}\right)\right]^{3} \times L^{2}\left(\widehat{\Omega}_{f}\right) \times\left[H^{1}\left(\widehat{\Omega}_{f}\right)\right]^{3} \times\left[H^{\frac{1}{2}}(\widehat{\Sigma})\right]^{3} \longrightarrow \\
\left(\left[H_{\widehat{\Sigma}}^{1}\left(\widehat{\Omega}_{f}\right)\right]^{3} \times L^{2}\left(\widehat{\Omega}_{f}\right) \times\left[L^{2}(\widehat{\Sigma})\right]^{3} \times\left[L^{2}\left(\widehat{\Omega}_{f}\right)\right]^{3}\right)^{\prime} .
\end{aligned}
$$

The same way, the linearized solid operator at state $\left(\widehat{\boldsymbol{d}}_{s}, \gamma\right) \in\left[H_{\Gamma_{D}}^{1}\left(\widehat{\Omega}_{s}\right)\right]^{3} \times$ $\left[L^{2}(\widehat{\Sigma})\right]^{3}$ is denoted by

$$
\mathrm{D} \mathcal{S}\left(\widehat{\boldsymbol{d}}_{s}, \gamma\right):\left[H_{\Gamma_{D}}^{1}\left(\widehat{\Omega}_{s}\right)\right]^{3} \times\left[H^{\frac{1}{2}}(\widehat{\Sigma})\right]^{3} \longrightarrow\left(\left[H_{\Gamma_{D} \cup \widehat{\Sigma}}^{1}\left(\widehat{\Omega}_{s}\right)\right]^{3} \times\left[L^{2}(\widehat{\Sigma})\right]^{3}\right)^{\prime} .
$$

And we finally introduce the linearized interface operator at state $\left(\widehat{\boldsymbol{u}}, \widehat{p}, \widehat{\boldsymbol{d}}_{f}, \widehat{\boldsymbol{d}}_{s}\right)$

$\mathrm{D} \mathcal{I}\left(\widehat{\boldsymbol{u}}, \widehat{p}, \widehat{\boldsymbol{d}}_{f}, \widehat{\boldsymbol{d}}_{s}\right):\left[H^{1}\left(\widehat{\Omega}_{f}\right)\right]^{3} \times L^{2}\left(\widehat{\Omega}_{f}\right) \times\left[H^{1}\left(\widehat{\Omega}_{f}\right)\right]^{3} \times\left[H^{1}\left(\widehat{\Omega}_{s}\right)\right]^{3} \longrightarrow\left[H^{-\frac{1}{2}}(\widehat{\Sigma})\right]^{3}$.

In terms of the operators introduced above, the tangent problem associated with [28] reads

$$
\left\{\begin{aligned}
\mathrm{D} \mathcal{F}\left(\widehat{\boldsymbol{u}}, \widehat{p}, \widehat{\boldsymbol{d}}_{f}, \gamma\right) \cdot\left(\delta \widehat{\boldsymbol{u}}, \delta \widehat{p}, \delta \widehat{\boldsymbol{d}}_{f}, \delta \gamma\right) & =-\mathcal{F}\left(\widehat{\boldsymbol{u}}, \widehat{p}, \widehat{\boldsymbol{d}}_{f}, \gamma\right) \\
\mathrm{D} \mathcal{S}\left(\widehat{\boldsymbol{d}}_{s}, \gamma\right) \cdot\left(\delta \widehat{\boldsymbol{d}}_{s}, \delta \gamma\right) & =-\mathcal{S}\left(\widehat{\boldsymbol{d}_{s}}, \gamma\right) \\
\mathrm{D} \mathcal{I}\left(\widehat{\boldsymbol{u}}, \widehat{p}, \widehat{\boldsymbol{d}}_{f}, \widehat{\boldsymbol{d}}_{s}\right) \cdot\left(\delta \widehat{\boldsymbol{u}}, \delta \widehat{p}, \delta \widehat{\boldsymbol{d}}_{f}, \delta \widehat{\boldsymbol{d}}_{s}\right) & =-\mathcal{I}\left(\widehat{\boldsymbol{u}}, \widehat{p}, \widehat{\boldsymbol{d}}_{f}, \widehat{\boldsymbol{d}}_{s}\right)
\end{aligned}\right.
$$

Once the linear fluid, solid and interface operators $\mathrm{D} \mathcal{F}, \mathrm{D} \mathcal{S}$ and $\mathrm{D} \mathcal{I}$ are defined, we can introduce the linear Steklov-Poincaré operators $S_{F, l}$ and $S_{S, l}$ using the formulae of Section 5.3 with the linearized operators instead of the nonlinear operators. It may be noted that the linear Steklov-Poincaré operators are different from the linearization of the nonlinear Steklov operators of Section 5.3.

\subsection{Implementation issues}

In this subsection, we briefly describe the general domain decomposition algorithm used to solve the linear problems introduced above, namely both the DirichletNeumann and the Neumann-Neumann algorithms (see Figures 3 and 4). Note that 
other choices for preconditioners are possible, e. g. using the Robin transmission conditions proposed by Badia et al. in (Badia et al., 2008a; Badia et al., 2009) for linear problems.

\subsubsection{General algorithm}

Following the practical implementation, we decompose the algorithm according to three distinct solvers: the master (which, roughly speaking, solves the third equation of [31] by a GMRES method), the fluid solver (which solves the first equation of [31]) and the solid solver (which solves the second equation of [31]).

The iterative algorithm is as follows:

1) Evaluate the Newton residual (right-hand sides of [31]).

2) Initialization of the Domain Decomposition method:

a) Lifting of the external load and boundary conditions, that is solve the first and second equations of [31] with $\delta \gamma=0$.

b) Computation of the right-hand side of the Schur complement by the master, insert the residuals received from the fluid and from the solid into the third equation of [31]. This step evaluates how far the solution with zero on the interface is from the true solution of the coupled problem [31].

c) Preconditioning the right-hand side of the Schur complement.

3) Iteration until convergence of the GMRES algorithm on the Schur complement by the master, which updates the displacement $\delta \gamma$, sends it to the fluid and solid solvers in order to

a) Evaluate the new residual

b) Preconditioning the residual

4) End of the domain decomposition algorithm.

The detailed description of these steps for both the Dirichlet-Neumann and Neumann-Neumann algorithms is given in Figures 3 and 4, which are commented in the following two paragraphs. Note that the steps [2a] and [4] do not depend on the preconditioner.

\subsubsection{Dirichlet-Neumann preconditioner (Figure 3)}

The Dirichlet-Neumann preconditioner amounts to preconditioning the Schur complement using the solid problem only, namely the exact tangent problem of the solid (second equation of [31]) with Neumann boundary conditions. It is worth noticing that the preconditioning step is not performed in parallel since only the structure problem is used to precondition the residual. For each iteration [3] of the GMRES algorithm on the Schur complement, the master sends $\delta \gamma$ in Step [3a] to the fluid solver only, which returns a residual to the master. In Step [3b], the master sends this residual to the solid solver, which applies the preconditioner and returns a displacement $\delta \tilde{\gamma}$. The master then sums the displacements $\frac{1}{2}(\delta \gamma+\delta \tilde{\gamma})$ and computes a new displace- 


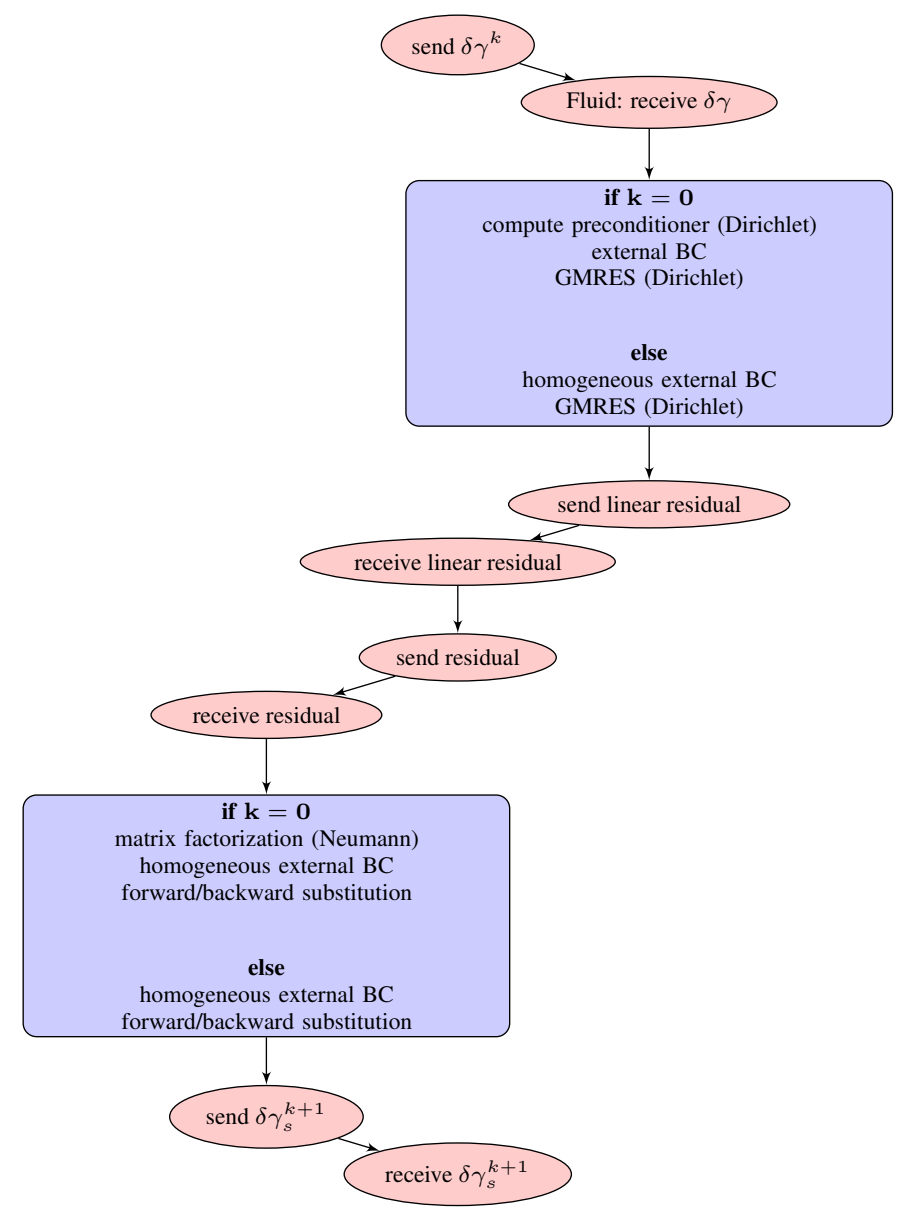

Figure 3. Detailed description of the domain decomposition algorithm: DirichletNeumann

ment using the update formula of the GMRES algorithm. At convergence, the final value of $\delta \gamma$ is known and the solutions in the fluid and in the solid can be computed.

Let us point out that the Dirichlet-Neumann algorithm described above is a purely sequential algorithm.

\subsubsection{Neumann-Neumann preconditioner (Figure 4)}

The Neumann-Neumann preconditioner uses both the tangent fluid problem and the tangent solid problem (first and second equations of [31]) with Neumann boundary conditions. This algorithm is fully parallel since both the preconditioning steps 


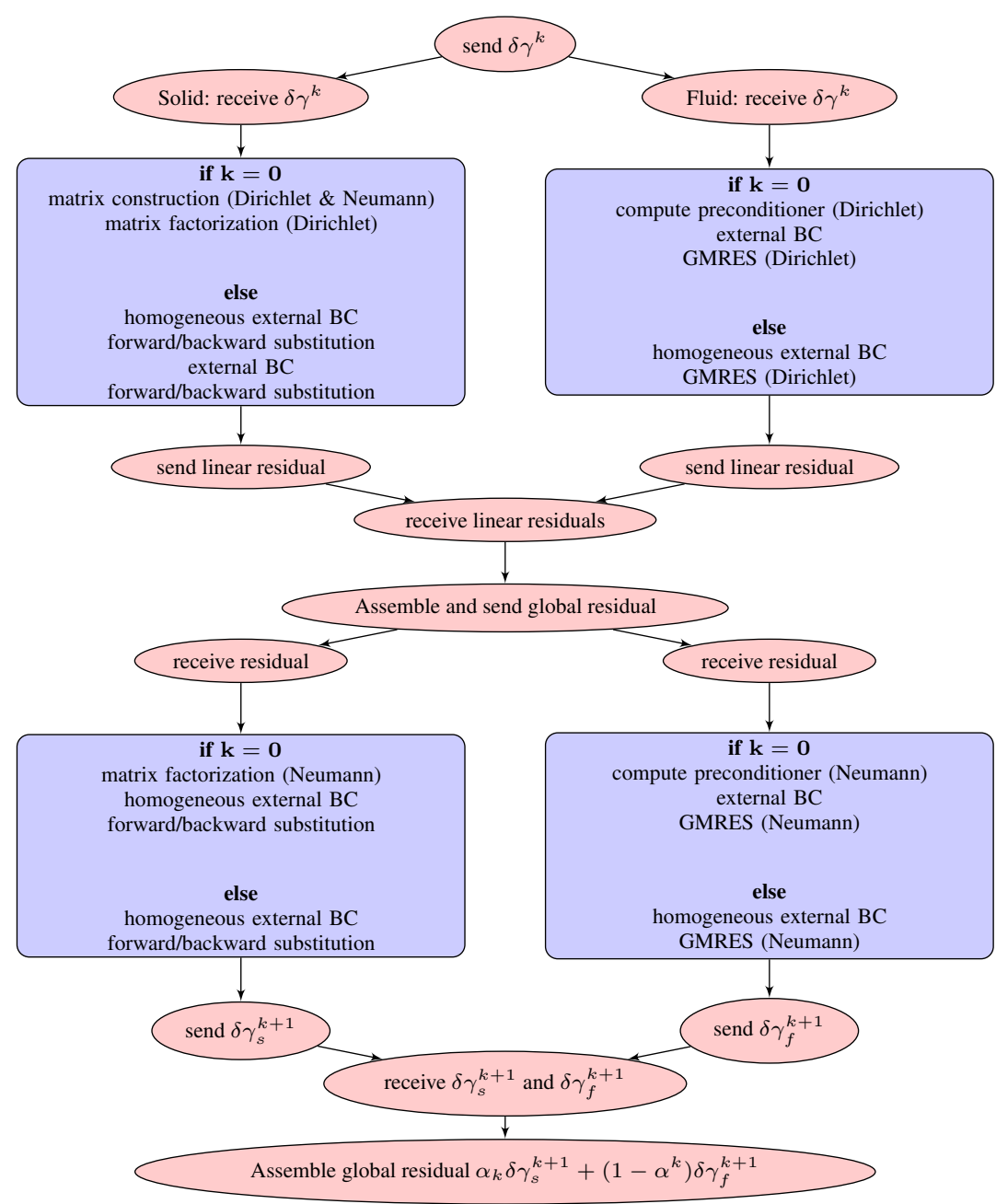

Figure 4. Detailed description of the domain decomposition algorithm: NeumannNeumann

[2c] and [3b] and residual evaluation steps [3a] can be done simultaneously by the fluid and solid solvers. Although for the tangent solid problem, considering Neumann boundary conditions is standard, for the tangent fluid problem this is not the case. In particular, shape derivative terms (that depend on the lifting $\boldsymbol{w}\left(\delta \widehat{\boldsymbol{d}}_{f}\right)$ of the fluid domain displacement, and thus on the solution $\delta \widehat{\boldsymbol{d}}_{f}$ itself on the interface) enter the stiffness matrix (see [35] in the Appendix) when Neumann boundary conditions are considered. Yet, the lifting matrix of [17] is never constructed and neither is the fluid 
tangent matrix. Therefore, each iteration of the GMRES algorithm to solve the tangent fluid problem requires the full solution of [17] by a GMRES algorithm. In practical implementation, it is easier and less expensive to neglect the shape derivatives terms in [35]. Doing so, we slightly modify the classical Neumann-Neumann preconditioner.

In order for the Neumann-Neumann preconditioner to be efficient, the weight associated with each subdomain (that is $\alpha^{k}$ ) should be such that the "effective stiffness" matrices (taking into account inertia and stiffness, and the $\alpha^{k}$ in [7] and Figure 4) of the fluid and structure are spectrally equivalent. In other words, the local effective stiffness at each degree of freedom on the interface should be of the same order for the fluid and the structure. To this aim, we follow (Le Tallec, 1994, Section 3.3, p. 155) and replace the scalar value $\alpha^{k}$ in [7] by a diagonal matrix where the coefficient $\alpha^{k}(i)$ depends on the degrees of freedom. In particular, denoting by $D S^{k}(i)$ and $D F^{k}(i)$ the diagonal elements of the matrices of [31] associated with the degree of freedom $i$ of the interface, for the solid and fluid respectively, we set $\alpha^{k}(i)=D S^{k}(i) /\left(D S^{k}(i)+D F^{k}(i)\right)$. This choice is optimal. As we will see in Section 7 , it has drastic effects in the case of fluid-structure interaction. Indeed the stiffness of the structure scales as $\delta t^{-2}$ whereas the stiffness of the fluid scales as $\delta t^{-1}$. Hence, we may expect the optimal Neumann-Neumann preconditioner to reduce to a Dirichlet-Neumann preconditioner (using the structure) as the time step vanishes. This is confirmed by the numerical tests.

\subsection{Complexity analysis}

Let us make a formal complexity analysis to have a rough hint on the cost of the Steklov type, Dirichlet to Neumann formulation based, and partitioned Newton type methods. We make the following assumptions: the fluid to be solved at each time step is linear (e.g. semi-implicit Euler scheme for Navier-Stokes equations), the structure problem is solved by a Newton algorithm and the linearized structure problems by direct methods. We only take into account the factorization for the resolution of the structure sub-problem and consider the matrices as already factorized when dealing with linear domain decomposition methods.

Let us recall that (I) is the algorithm introduced in Section 6 (that is the domain decomposition method applied on the linearized global problem), (II) refers to the Newton method on the symmetric Steklov formulation, and (III), to the Newton method on the DtoN formulation.

In the following analysis we assume that the number of Newton iterations, $\widetilde{N}_{F S I}$, for the global problem in formulations (II) and (III) is the same. Let $N_{F S I}$ denote the number of Newton iterations for the formulation (I). We denote by $N_{s}$ the number of iterations for a Newton algorithm in the structure problem. The number of GMRES iterations $G$ is assumed not to depend on the algorithm if optimal preconditioners (let say Dirichlet-Neumann) are used. In the sequel $\mathrm{Cr}$ and $\mathrm{Fa}$ denote respectively the cost of the construction and factorization of a matrix in the solid, $F l_{1}$ the resolution 
cost per time step of the fluid problem, and $\mathrm{Fl}_{2}$ the resolution cost for a tangent fluid problem. The estimations of costs for the three types of methods are gathered in Table 1 both for a sequential and a parallel implementation when possible. For the parallel implementation, we have assumed that $\mathrm{Fa}+\mathrm{Cr} \geq \mathrm{Fl}$ and $\mathrm{Fl} \geq \mathrm{Fa}$.

Table 1. Estimation of the computational cost

\begin{tabular}{|c|c|c|c|}
\hline Method & (I) preconditioned & (II) preconditioned & (III) Newton on \\
& partitioned Newton & Newton on Steklov & DtoN-formulation \\
\hline Sequential & $N_{F S I}[2 F a+C r$ & $\widetilde{N}_{F S I}\left[\left(N_{s}+1\right)(F a+C r)\right.$ & $\widetilde{N}_{F S I}\left[\left(N_{s}+1\right)(F a+C r)\right.$ \\
& $\left.+G F l_{2}+F l_{1}\right]$ & $\left.+F a+G F l_{2}+F l_{1}\right]$ & $\left.+F l_{1}+G F l_{2}\right]$ \\
\hline Parallel & $N_{F S I}[2 F a+C r$ & $\widetilde{N}_{F S I}\left[\left(N_{s}+1\right)(F a+C r)\right.$ & - \\
& $\left.+G F l_{2}\right]$ & $\left.+F a+G F l_{2}\right]$ & \\
\hline
\end{tabular}

Let us comment on Table 1. For the sequential implementation the estimations for the method (II) and (III) only differ by the factorization cost of a solid tangent matrix, which is rather small with respect to the whole cost. This is in agreement with the tests performed in (Deparis et al., 2006) where method (II) is shown to be roughly equivalent to method (III) in terms of cost. If $N_{F S I} \approx \widetilde{N}_{F S I}$, method (I) should be at least as efficient as the first two, especially if the structure is nonlinear and expensive. On the contrary, if $\mathrm{Fl} \geq \mathrm{Fa}+\mathrm{Cr}$ then the parallel implementations of methods (I) and (II) seem to be completely equivalent in terms of cost, which is only determined by the fluid. For the parallel implementation, the cost reduction strongly depends on the number of GMRES iterations, and the method (I) still seems to compete with method (II). Note that, if $N_{F S I}>\widetilde{N}_{F S I}$, method (I) may lose efficiency with respect to methods (II) and (III).

The condition $F a+C r \geq F l$ is almost never satisfied if standard shell elements are used. However, this condition may be satisfied when 3D shell elements are used to model more realistic constitutive laws for the structure (see Section 4). Let us consider for instance a mesh with 38000 nodes in the fluid (let say 150000 degrees of freedom). For MITC4 shell elements, we then have 3300 nodes and 16500 degrees of freedom. Numerical tests show that in this case, with the same computer, $F l \simeq 45 \mathrm{~s}, F a \simeq 0.7 \mathrm{~s}$ and $C r \simeq 1.7 \mathrm{~s}$. Let us now consider 3D shell elements (hexahedra, 27 nodes per element) on the same mesh. The number of nodes for the structure increases from 3300 to 22100 , and the number of degrees of freedom from 16500 to 66300 . The costs for the solid are now $F a \simeq 13 s$ and $C r \simeq 50 s$. We are thus in the situation $C r+F a \geq F l$ and $F l \geq F a$.

\section{Numerical tests}

In this section we illustrate the behavior of the linear Domain Decomposition method (I), with Dirichlet-Neumann and Neumann-Neumann preconditioners, by performing some numerical simulations. As regards efficiency, we make some comparisons with the nonlinear Domain Decomposition method (III), reported on in (Fernández et al., 2005). 
In all the computations the structure is modeled by 3D shell elements, as reported in Section 4.2, with a neo-Hookean constitutive law in finite deformation. For the space discretization we use a $\mathbb{Q}_{2}$-finite element (27 nodes) combined with a MITC interpolation rule in the thin direction of the hexaedra. This allows us to deal with three dimensional constitutive laws, and several layers of elements (from one to three in the tests). A mid-point rule is used for the time discretization. For the fluid, we consider the Navier-Stokes equation with an ALE formulation [11]. The fluid equations are discretized in space using $\mathbb{P}_{1} / \mathbb{P}_{1}$-SUPG-stabilized finite elements, and in time by a semi-implicit backward-Euler scheme.

\subsection{Flow in a compliant straight tube}

We consider here the benchmark test described in (Formaggia et al., 2001). The fluid computational domain is a cylinder of radius $R_{0}=0.5 \mathrm{~cm}$ and of length $L=$ $5 \mathrm{~cm}$. The tube wall has a thickness $h=0.1 \mathrm{~cm}$ and the rest of physical parameters are $E=3 \cdot 10^{6}$ dynes $/ \mathrm{cm}^{2}, \nu=0.3$ and $\rho_{s}=1.2 \mathrm{~g} / \mathrm{cm}^{3}$. For the fluid we have $\mu=0.035$ poise and $\rho_{f}=1 \mathrm{~g} / \mathrm{cm}^{3}$. The numerical computations are performed using a fluid mesh with 38400 tetrahedra and a solid mesh with one to three layers (of 160 hexahedra each), the time step size is $\delta t=10^{-4} \mathrm{~s}$.

Initially, the fluid is at rest and an over pressure of $1.3332 \cdot 10^{4}$ dynes $/ \mathrm{cm}^{2}$ $(10 \mathrm{mmHg})$ is imposed at the inlet boundary during $0.005 \mathrm{~s}$. The results consist of a pressure wave propagation and is comparable with those obtained with more standard shell elements (see e.g. (Gerbeau et al., 2003; Fernández et al., 2007) with the MITC4 shell element).

The same numerical computation has been carried out using method (III). A comparison of the efficiency of both methods, in terms of the number of layers of 3D shell elements in the structure, is reported on in Table 2. We observe that, with a mono- or bi-layered structure, method (III) is slightly faster than method (I), mainly due to the reduced number of Newton iterations (see Section 6.3). With a three-layered structure, however, the Newton algorithm of method (III) fails to converge. Method (I) remains practically insensitive to the numbers of layers. This shows that the complexity of the solid problem might compromise the effectiveness of Newton's method when applied to the non-linear Dirichlet-to-Neumann formulation, whereas method (I) is robust with respect to the number of layers.

Note that the estimates of Table 1 are in good agreement with the results of Table 2. Method (I) is outperformed by method (III) for 1 layer because of the number of Newton iterations: $N_{F S I}=5>\widetilde{N}_{F S I}=2$.

\subsubsection{Sensitivity to the added-mass effect}

In this paragraph we investigate the impact of the added-mass effect on the effectiveness of the Dirichlet-Neumann and Neumann-Neumann preconditioners. 
Table 2. Efficiency over 10 time steps: dimensionless CPU time and mean number of GMRES and Newton iterations (symbol $\times$ indicates that the overall algorithm failed to converge)

\begin{tabular}{|l|c|c|c|c|c|c|}
\hline Number of solid layers & \multicolumn{2}{|c|}{1} & \multicolumn{2}{|c|}{2} & \multicolumn{2}{|c|}{3} \\
\hline Method & (I) & (III) & (I) & (III) & (I) & (III) \\
\hline CPU time & 1.2 & 1 & 1.7 & 1.4 & 2.2 & $\times$ \\
\hline GMRES iterations & 8.1 & 10.7 & 8.5 & 10.6 & 8.6 & $\times$ \\
\hline Newton iterations & 4.7 & 2.6 & 4.7 & 2.8 & 4.4 & $\times$ \\
\hline
\end{tabular}

Table 3. Average number of GMRES iterations for the Dirichlet-Neumann (DD) and Neumann-Neumann $(N N)$, with $\alpha^{k}=1 / 2$, preconditioners in terms of the tube length $L$

\begin{tabular}{|c||c|c|c|}
\hline Prec. & $5(\mathrm{~cm})$ & $10(\mathrm{~cm})$ & $15(\mathrm{~cm})$ \\
\hline \hline DN & 8.5 & 11.9 & 14.5 \\
\hline NN & 13.0 & 13.7 & 14.8 \\
\hline
\end{tabular}

Table 4. Average number of GMRES iterations for the Dirichlet-Neumann (DN) and Neumann-Neumann $(N N)$, with $\alpha^{k}=1 / 2$, preconditioners in terms of the time-step length $\delta t$

\begin{tabular}{|c||c|c|c|c|}
\hline Prec. & $10^{-3}(\mathrm{~s})$ & $0.5 \times 10^{-3}(\mathrm{~s})$ & $10^{-4}(\mathrm{~s})$ & $0.5 \times 10^{-4}(\mathrm{~s})$ \\
\hline \hline $\mathrm{DN}$ & 5.8 & 6.7 & 8.5 & 9.2 \\
\hline $\mathrm{NN}$ & 48.5 & 27.3 & 8.5 & 7.3 \\
\hline
\end{tabular}

We first consider the case in which the Neumann-Neumann preconditioner is used with the homogeneous scaling $\alpha^{k}=1 / 2$. Table 3 reports on the average number of GMRES interations (over 10 time steps) for differents values of the domain length $L$. We observe that for both preconditioners the number of iterations increases with $L$. The sensitivity being slightly bigger for Dirichlet-Neumann, but with a reduced number of iterations. In Table 4 we present the sensitivity with respect to the time step length $\delta t$. We observe that the Dirichlet-Neumann and Neumann-Neumann preconditioners show a complete opposite behavior. For the Dirichlet-Neumann preconditioner the number of GMRES iterations increases by reducing the time step length, whereas for Neumann-Neumann the number of GMRES iterations increases with $\delta t$. Note that increasing the time step length is dramatic for the Neumann-Neumann pre- 
conditioner. This poor behavior is due to the unphysical balance of the scaling parameters $\alpha^{k}=1 / 2$, as shown by the results reported in Table 5, using the heterogeneous Neumann-Neumann scaling discussed in Section 6.2. These results also show that, with this automatic scaling and for the physical problem under consideration, the Neumann-Neumann preconditioner becomes a Dirichlet-Neumann preconditioner.

Table 5. Average number of GMRES iterations for the Dirichlet-Neumann (DN) and Neumann-Neumann (NN), with the heterogeneous scaling (see Section 6.2), preconditioners in terms of the time-step length $\delta t$

\begin{tabular}{|c||c|c|c|c|}
\hline Prec. & $10^{-3}(\mathrm{~s})$ & $0.5 \times 10^{-3}(\mathrm{~s})$ & $10^{-4}(\mathrm{~s})$ & $0.5 \times 10^{-4}(\mathrm{~s})$ \\
\hline \hline $\mathrm{DN}$ & 5.8 & 6.7 & 8.5 & 9.2 \\
\hline $\mathrm{NN}$ & 5.8 & 6.7 & 8.5 & 9.2 \\
\hline
\end{tabular}

\subsection{Flow in a compliant vessel with an aneurysm}

We now consider the FSI numerical results reported on in (Salsac et al., 2005) using in vitro aneurysm geometries. The fluid computational domain is represented in Figure 5. The geometry correspond to the mock-up of a middle-size aneurysm with a dilatation ratio $D / d=2.4$ (maximum diameter to inlet diameter ratio) and aspect ratio $L / d=3.9$ (length to inlet diameter ratio), with $d=1.7 \mathrm{~cm}$ (we refer to (Salsac et al., 2005; Salsac et al., 2006) for the details). The wall has a uniform thickness $h=$ $0.17 \mathrm{~cm}$ and the physical parameters are given by $E=6 \cdot 10^{6}$ dynes $/ \mathrm{cm}^{2}, \nu=0.3$ and $\rho_{s}=1.2 \mathrm{~g} / \mathrm{cm}^{3}$. For the fluid we have $\mu=0.035$ poise and $\rho_{f}=1 \mathrm{~g} / \mathrm{cm}^{2}$. The fluid and solid meshes are made of 165888 tetrahedra and 640 hexahedra, respectively. The time step size is $\delta t=1.68 \times 10^{-3} \mathrm{~s}$.

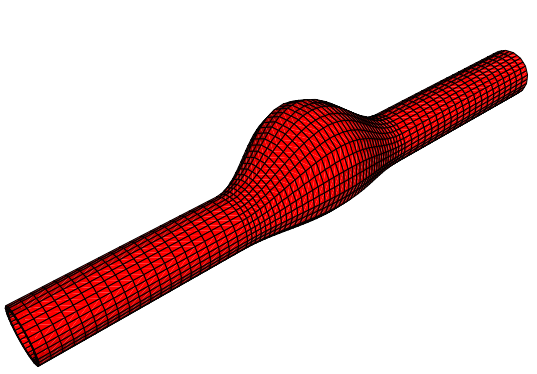

(a)

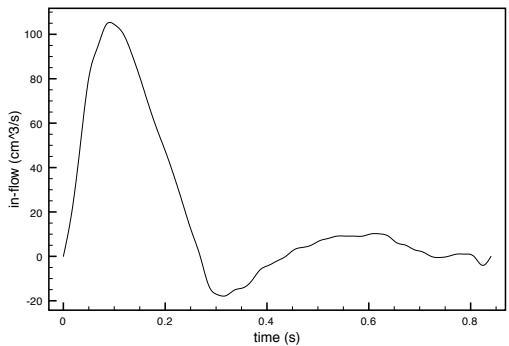

(b)

Figure 5. Aneurysm geometry (a) and in-flow rate data (b)

Initially, the fluid is at rest. The in-flow rate corresponding to a cardiac cycle, see Figure 5(right), is imposed on the inlet boundary. A resistive-like bound- 


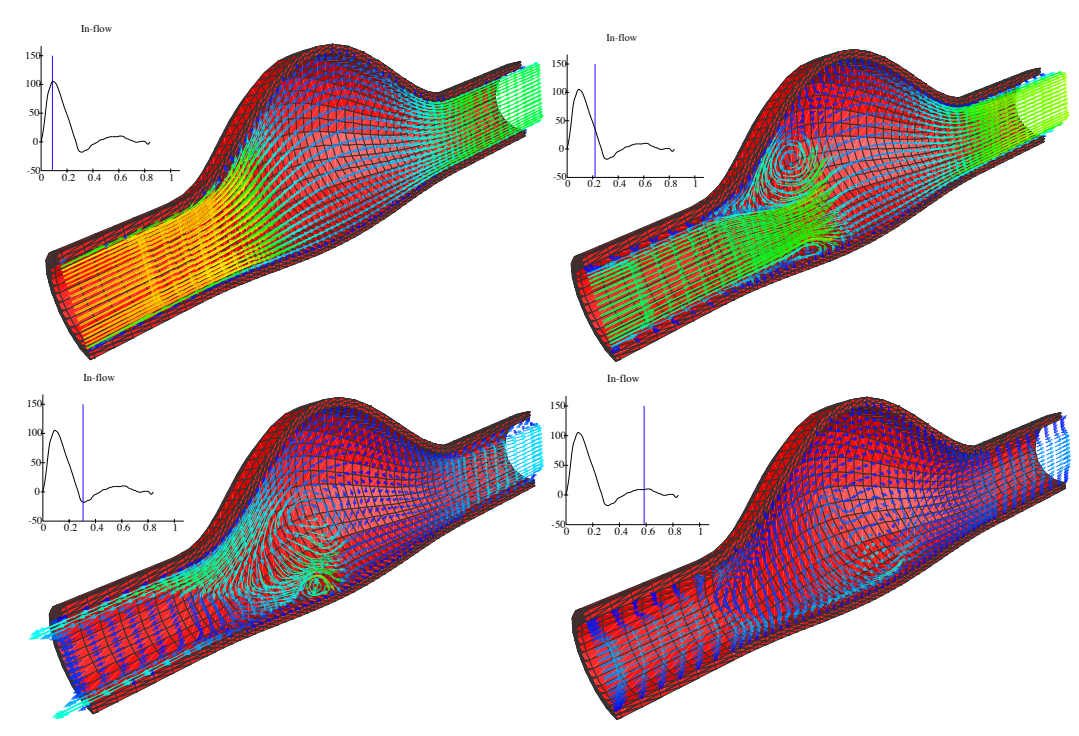

Figure 6. Deformation of the structure and fluid velocity field at time $87.36 \times 10^{-3}$, $218.4 \times 10^{-3}, 305.76 \times 10^{-3}$ and $584.64 \times 10^{-3} s$

ary condition is prescribed on the outlet boundary, the value of the resistance being $R=3 \times 10^{3}$ dyne $/ \mathrm{cm}^{3}$. In Figure 6 we have reported on some snapshots of the wall deformation and the fluid velocity field at different time instants. These results are in agreement with those obtained with the MITC4 shell element in (Salsac et al., 2005).

As in the previous experiment, the same numerical simulation has been performed using method (III). Once again, method (III) performs slightly faster. Nevertheless, the efficiency gap between both methods has been reduced, due to the increasing in the computational cost of the structural solver.

\section{Conclusion}

We have proposed a Newton algorithm for fluid-structure problems. The starting point of the method is the same as for the so-called monolithic approaches since we consider the global fluid-structure equations, but the tangent problem is solved with domain decomposition techniques. The resulting method is therefore partitioned: it is based on two different solvers for the fluid and the structures and can be parallelized. As a side remark of this study, we have noticed that the Neumann-Neumann preconditioner actually behaves like a Dirichlet-Neumann preconditioner for this kind of FSI problems, as soon as the Schur complements of the fluid and the solid are correctly balanced. 
The proposed approach does not outperform usual partitioned Newton methods in various test cases. This fact has been explained through a simplified complexity analysis which showed that the scheme is expected to reach optimal performance when the structure is expensive. Nevertheless, for more complex structure models, like multi-layered shells, our numerical tests have shown that our approach can be actually more efficient and robust than usual nonlinear partitioned schemes.

\section{References}

Badia S., Nobile F., Vergara C., " Fluid-structure partitioned procedures based on Robin transmission conditions”, J. Comp. Phys., vol. 227, p. 7027-7051, 2008 a.

Badia S., Nobile F., Vergara C., " Robin-Robin preconditioned Krylov methods for fluidstructure interaction problems”, Comput. Methods Appl. Mech. Engrg., vol. 198, n 33-36, p. 2768-2784, 2009.

Badia S., Quaini A., Quarteroni A., “ Modular vs. non-modular preconditioners for fluidstructure systems with large added-mass effect”, Comput. Methods Appl. Mech. Engrg., vol. 197, n 49-50, p. 4216-4232, 2008b.

Bathe K., Zhang H., " Finite element developments for general fluid flows with structural interactions”, Int. J. Num. Meth. Engng., 2004.

Batina J., "Unsteady Euler airfoil solutions using unstructured dynamic meshes", AIAA J., vol. 28, n 8, p. 1381-1388, 1990.

Bazilevs Y., Calo V., Hughes T., Zhang Y., Isogeometric Fluid-Structure Interaction: Theory, Algorithms, and Computations, Technical Report n 08-16, ICES, 2008.

Bazilevs Y., Calo V., Zhang Y., Hughes T., “ Isogeometric fluid-structure interaction analysis with applications to arterial blood flow", Comput. Mech., vol. 38, p. 310-322, 2006.

Burman E., Fernández M., " Stabilization of explicit coupling in fluid-structure interaction involving fluid incompressibility", Comput. Methods Appl. Mech. Engrg., vol. 198, n 5-8, p. 766-784, 2009.

Caillerie D., Mourad A., A. R., “ Cell-to-muscle homogenization. Application to a constitutive law for the myocardium”, Math. Model. Num. Anal., vol. 37, p. 681-698, 2003.

Causin P., Gerbeau J.-F., Nobile F., “ Added-mass effect in the design of partitioned algorithms for fluid-structure problems”, Comp. Meth. Appl. Mech. Engng., vol. 194, n 42-44, p. 45064527, 2005.

Chapelle D., Bathe K., The Finite Element Analysis of Shells - Fundamentals, Springer Verlag, 2003a.

Chapelle D., Ferent A., " Modeling of the inclusion of a reinforcing sheet within a 3D medium", Math. Models Methods Appl. Sci., vol. 13, n 4, p. 573-595, 2003b.

Chapelle D., Ferent A., Bathe K., " 3D-shell elements and their underlying mathematical model”, Math. Models Methods Appl. Sci., vol. 14, n 1, p. 105-142, 2004.

Chapelle D., Ferent A., Le Tallec P., " The treatment of "pinching locking" in 3D-shell elements", M2AN Math. Model. Numer. Anal., vol. 37, n 1, p. 143-158, $2003 \mathrm{c}$.

Deparis S., Numerical Analysis of Axisymmetric Flows and Methods for Fluid-Structure Interaction Arising in Blood Flow Simulation, PhD thesis, EPFL, Switzerland, 2004. 
Deparis S., Discacciati M., Fourestey G., Quarteroni A., “ Fluid-structure algorithms based on Steklov-Poincaré operators", Comput. Methods Appl. Mech. Engrg., vol. 195, n 41-43, p. 5797-5812, 2006.

Deparis S., Discacciati M., Quarteroni A., “ A domain decomposition framework for fluidstructure interaction problems", Proceedings of the Third International Conference on Computational Fluid Dynamics (ICCFD3), 2004.

Dettmer W., Perić D., “ A computational framework for fluid-structure interaction: Finite element formulation and applications", Comp. Meth. Appl. Mech. Engrg., vol. 195, n 41-43, p. 5754-5779, 2006.

Donéa J., Giuliani S., Halleux J. P., “ An arbitrary Lagrangian-Eulerian finite element method for transient dynamic fluid-structure interactions", Comp. Meth. Appl. Mech. Engng., vol. 33, p. 689-723, 1982.

Farhat C., van der Zee K., Geuzaine P., “ Provably second-order time-accurate loosely-coupled solution algorithms for transient nonlinear aeroelasticity", Comp. Meth. Appl. Mech. Engng., vol. 195, n $\mathrm{n}^{\circ}$ 17-18, p. 1973-2001, 2006.

Felippa C., Park K., “ Staggered transient analysis procedures for coupled mechanical systems: formulation", Computer Methods in Applied Mechanics and Engineering, vol. 24, $\mathrm{n}^{\circ} 1$, p. 61-111, 1980.

Felippa C., Park K., Farhat C., "Partitioned analysis of coupled mechanical systems”, Computer methods in applied mechanics and engineering, vol. 190, n²4-25, p. 3247-3270, 2001.

Fernández M., Gerbeau J.-F., Grandmont C., “ A projection semi-implicit scheme for the coupling of an elastic structure with an incompressible fluid", Internat. J. Numer. Methods Engrg., vol. 69, n 4, p. 794-821, 2007.

Fernández M., Moubachir M., “ A Newton method using exact Jacobians for solving fluidstructure coupling”, Comp. \& Struct., vol. 83, p. 127-142, 2005.

Formaggia L., Gerbeau J.-F., Nobile F., Quarteroni A., " On the Coupling of 3D and 1D NavierStokes equations for Flow Problems in Compliant Vessels", Comp. Meth. Appl. Mech. Engrg., vol. 191, n 6-7, p. 561-582, 2001.

Förster C., Wall W., Ramm E., “ Artificial Added Mass Instabilities in Sequential Staggered Coupling of Nonlinear Structures and Incompressible Flows", Comp. Meth. Appl. Mech. Engrg., vol. 196, p. 1278-1293, 2006.

Fung Y., Fronek K., Patitucci P., " Pseudoelasticity of arteries and the choice of its mathematical expression”, Am. J. Physiol., vol. 237, p. 620-631, 1979.

Gee M., Küttler U., Wall W., “ Truly monolithic algebraic multigrid for fluid-structure interaction”, Int. J. Numer. Meth. Engng., 2010. DOI: 10.1002/nme.3001.

Gerbeau J.-F., Vidrascu M., “ A quasi-Newton algorithm based on a reduced model for fluidstructure interactions problems in blood flows", Math. Model. Num. Anal., vol. 37, n 4, p. 631-648, 2003.

Gerbeau J.-F., Vidrascu M., Frey P., " Fluid-Structure Interaction in Blood Flows on Geometries based on Medical Imaging”, Comp. \& Struct., vol. 83, n²-3, p. 155-165, 2005.

Guidoboni G., Glowinski R., Cavallini N., Canic S., " Stable loosely-coupled-type algorithm for fluid-structure interaction in blood flow", Journal of Computational Physics, vol. 228, $\mathrm{n}^{\circ} 18$, p. 6916-6937, 2009. 
Heil M., " An efficient solver for the fully coupled solution of large-displacement fluid-structure interaction problems", Comput. Methods Appl. Mech. Engrg., vol. 193, n 1-2, p. 1-23, 2004.

Heil M., Hazel A., Boyle J., " Solvers for large-displacement fluid-structure interaction problems: Segregated vs. monolithic approaches”, Comp. Mech., vol. 43, p. 91-101, 2008.

Holzapfel A., Gasser T., R.W. O., “ A new constitutive framework for arterial wall mechanics and a comparative study of material models", J. Elasticity, vol. 61, p. 1-48, 2000.

Hron J., Turek S., “ A monolithic FEM/Multigrid solver for an ALE formulation of fluidstructure interaction with applications in biomechanics", Fluid-structure interaction - modelling, simulation, optimisation, Computational Science and Engineering, vol. 53, Springer, p. 146-170, 2006.

Hübner B., Walhorn E., Dinkle D., “ A monolithic approach to fluid-structure interaction using space-time finite elements”, Comp. Meth. Appl. Mech. Engng., vol. 193, p. 2087-2104, 2004.

Küttler U., Gee M., Förster C., Comerford A., Wall W., " Coupling strategies for biomedical fluid-structure interaction problems", Int. J. Numer. Meth. Biomed. Engng., vol. 26, n’ 3-4, p. 305-321, 2009.

Küttler U., Wall W., "Fixed-point fluid-structure interaction solvers with dynamic relaxation”, Comp. Mech., vol. 43, n 1, p. 61-72, 2008.

Le Tallec P., " Domain decomposition methods in computational mechanics”, Computational Mechanics Advances, Vol. 1, no.2, North Holland, p. 123-217, 1994.

Le Tallec P., Mouro J., " Fluid structure interaction with large structural displacements", Comput. Meth. Appl. Mech. Engrg., vol. 190, p. 3039-3067, 2001.

Matthies H., Steindorf J., " Partitioned but strongly coupled iteration schemes for nonlinear fluid-structure interaction”, Comp. \& Struct., vol. 80, n²7-30, p. 1991-1999, 2002.

Matthies H., Steindorf J., " Partitioned strong coupling algorithms for fluid-structure interaction”, Comp. \& Struct., vol. 81, p. 805-812, 2003.

Michler C., van Brummelen E., de Borst R., “ An interface Newton-Krylov solver for fluidstructure interaction”, Int. J. Num. Meth. Fluids, vol. 47, n 10-11, p. 1189-1195, 2005.

Michler C., van Brummelen E., de Borst R., " Error-amplification analysis of subiterationpreconditioned GMRES for fluid-structure interaction", Comp. Meth. Appl. Mech. Engng., vol. 195, p. 2124-2148, 2006.

Mok D. P., Wall W. A., " Partitioned analysis schemes for the transient interaction of incompressible flows and nonlinear flexible structures", in K. S. W.A. Wall, K.U. Bletzinger (ed.), Trends in computational structural mechanics, CIMNE, Barcelona, 2001a.

Mok D. P., Wall W. A., Ramm E., " Partitioned analysis approach for the transient, coupled response of viscous fluids and flexible structures", in W. Wunderlich (ed.), Proceedings of the European Conference on Computational Mechanics, ECCM'99, TU Munich, 1999.

Mok D. P., Wall W. A., Ramm E., “ Accelerated iterative substructuring schemes for instationary fluid-structure interaction", in K. Bathe (ed.), Computational Fluid and Solid Mechanics, Elsevier, p. 1325-1328, 2001b.

Nobile F., Numerical approximation of fluid-structure interaction problems with application to haemodynamics, PhD thesis, EPFL, Switzerland, 2001.

Oijen C. H. v., Mechanics and design of fiber-reinforced vascular prostheses, $\mathrm{PhD}$ thesis, Technische Universiteit Eindhoven, 2003. 
Park K., Felippa C., DeRuntz J., “ Stabilization of staggered solution procedures for fluidstructure interaction analysis", Computational methods for fluid-structure interaction problems, p. 95-124, 1977.

Piperno S., Farhat C., Larrouturou B., " Partitioned procedures for the transient solution of coupled aeroelastic problems. Part I: Model problem, theory and two-dimensional application", Comp. Meth. Appl. Mech. Engrg., vol. 124, p. 79-112, 1995.

Quaini A., Quarteroni A., “ A semi-implicit approach for fluid-structure interaction based on an algebraic fractional step method", Math. Models Methods Appl. Sci., vol. 17, n 6, p. 957983, 2007.

Rugonyi S., Bathe K., " On finite element analysis of fluid flows coupled with structural interaction”, CMES - Comp. Modeling Eng. Sci., vol. 2, n 2, p. 195-212, 2001.

Salsac A.-V., Fernández M., Chomaz J., Le Tallec P., "Effects of the flexibility of the arterial wall on the wall shear stresses and wall tension in abdominal aortic aneurysms", Bulletin of the American Physical Society, 2005.

Salsac A.-V., Sparks S., Chomaz J., Lasheras J., “ Evolution of the wall shear stresses during the progressive enlargement of symmetric abdominal aortic aneurysms", J. Fluid Mech., vol. 550, p. 19-51, 2006.

Tezduyar T., " Finite Element Methods for Fluid Dynamics with Moving Boundaries and Interfaces", Arch. Comput. Methods Engrg., vol. 8, p. 83-130, 2001.

Tezduyar T., Sathe S., Keedy R., Stein K., "Space-time finite element techniques for computation of fluid-structure interactions", Comput. Meth. Appl. Mech. Engng., vol. 195, p. 20022027, 2006.

Thomas P., Lombard C., " Geometric conservation law and its application to flow computations on moving grids", AIAA J., vol. 17, n 10, p. 1030-1037, 1979.

van Brummelen E., " Added mass effects of compressible and incompressible flows in fluidstructure interaction", Journal of Applied Mechanics, vol. 76, n 2, p. 021206-07, 2009.

van Brummelen E., van der Zee K., de Borst R., “ Space/time multigrid for a fluid-structure interaction problem”, Applied Numerical Mathematics, vol. 58, n 12, p. 1951-1971, 2008.

van der Zee K., van Brummelen E., Akkerman I., de Borst R., “ Goal-oriented error estimation and adaptivity for fluid-structure interaction using exact linearized adjoint", 2010, to appear in Comp. Meth. Appl. Mech. Engng.

Vierendeels J., " Implicit coupling of partioned fluid-structure interaction solvers using reduced order models", in M. S. H.J. Bungartz (ed.), Fluid-Structure interaction, Modelling, Simulation, Optimization, Springer, p. 1-18, 2006.

Zhang H., Zhang X., Ji S., Guo Y. Ledezma G., Elabbasi N., deCougny H., “ Recent development of fluid-structure interaction capabilities in the ADINA system", Computers \& Structures, vol. 81, n 8-11, p. 1071-1085, 2003. 


\section{Appendix}

\section{A. Derivation of the weak formulation [18]}

Let $\left(\widehat{\boldsymbol{v}_{f}}, \widehat{q}\right) \in\left[H^{1}\left(\widehat{\Omega}_{f}\right)\right]^{3} \times L^{2}\left(\widehat{\Omega}_{f}\right)$, multiplying the fluid problem [11] by $\left(\boldsymbol{v}_{f}, q\right)=\left(\widehat{\boldsymbol{v}}_{f} \circ \widehat{\mathcal{A}}_{t}^{-1}, \widehat{q} \circ \widehat{\mathcal{A}}_{t}^{-1}\right)$ integrating over $\Omega_{f}\left(\widehat{\boldsymbol{d}}_{f}\right)$ and after integrations by parts we get

$$
\begin{gathered}
\frac{\mathrm{d}}{\mathrm{dt}} \int_{\Omega_{f}\left(\widehat{\boldsymbol{d}_{f}}\right)} \rho_{f} \boldsymbol{u} \cdot \boldsymbol{v}_{f} \mathrm{~d} \boldsymbol{x}+\int_{\Omega_{f}\left(\widehat{\boldsymbol{d}_{f}}\right)} \operatorname{div}\left[\rho_{f} \boldsymbol{u} \otimes\left(\boldsymbol{u}-\boldsymbol{w}\left(\widehat{\boldsymbol{d}_{f}}\right)\right)\right] \cdot \boldsymbol{v}_{f} \mathrm{~d} \boldsymbol{x} \\
+\int_{\Omega_{f}\left(\widehat{\boldsymbol{d}_{f}}\right)} \boldsymbol{\sigma}(\boldsymbol{u}, p): \nabla \boldsymbol{v}_{f} \mathrm{~d} \boldsymbol{x}-\int_{\Sigma\left(\widehat{\boldsymbol{d}_{f}}\right)} \boldsymbol{\sigma}(\boldsymbol{u}, p) \cdot \boldsymbol{v}_{f} \cdot \boldsymbol{n} \\
\mathrm{d} \boldsymbol{a}-\int_{\Gamma_{\mathrm{in}-\text { out }}} \boldsymbol{g} \cdot \boldsymbol{v}_{f} \mathrm{~d} \boldsymbol{a}-\int_{\Omega_{f}\left(\widehat{\boldsymbol{d}_{f}}\right)} q \operatorname{div} \boldsymbol{u} \mathrm{d} \boldsymbol{x}=0,
\end{gathered}
$$

where

$$
\boldsymbol{w}\left(\widehat{\boldsymbol{d}_{f}}\right)=\frac{\partial \widehat{\boldsymbol{d}_{f}}}{\partial t} \circ \widehat{\mathcal{A}}_{t}^{-1}
$$

For the structure, multiplying [12] by $\widehat{\boldsymbol{v}}_{s} \in\left[H_{\Gamma_{D}}^{1}\left(\widehat{\Omega}_{s}\right)\right]^{3}$, integrating by parts over $\widehat{\Omega}_{s}$, one gets

$\int_{\widehat{\Omega}_{s}} \rho_{0} \frac{\partial^{2} \widehat{\boldsymbol{d}_{s}}}{\partial t^{2}} \cdot \widehat{\boldsymbol{v}_{s}} \mathrm{~d} \hat{\boldsymbol{x}}+\int_{\widehat{\Omega}_{s}} \frac{\partial W}{\partial F}\left(\mathrm{I}+\nabla \widehat{\boldsymbol{d}_{s}}\right): \nabla \widehat{\boldsymbol{v}_{s}} \mathrm{~d} \hat{\boldsymbol{x}}-\int_{\widehat{\Sigma}} \frac{\partial W}{\partial F}\left(\mathrm{I}+\nabla \widehat{\boldsymbol{d}_{s}}\right) \widehat{\boldsymbol{n}}_{s} \cdot \widehat{\boldsymbol{v}_{s}} \mathrm{~d} \hat{\boldsymbol{a}}=0$,

where $\rho_{0}=\widehat{J}_{s} \rho_{s}$. Therefore, taking into account the coupling condition [16], it follows that

$$
\begin{array}{r}
\frac{\mathrm{d}}{\mathrm{dt}} \int_{\Omega_{f}\left(\widehat{\boldsymbol{d}_{f}}\right)} \rho_{f} \boldsymbol{u} \cdot \boldsymbol{v}_{f} \mathrm{~d} \boldsymbol{x}+\int_{\Omega_{f}\left(\widehat{\boldsymbol{d}_{f}}\right)} \operatorname{div}\left[\rho_{f} \boldsymbol{u} \otimes\left(\boldsymbol{u}-\boldsymbol{w}\left(\widehat{\boldsymbol{d}_{f}}\right)\right)\right] \cdot \boldsymbol{v}_{f} \mathrm{~d} \boldsymbol{x} \\
+\int_{\Omega_{f}\left(\widehat{\boldsymbol{d}_{f}}\right)} \boldsymbol{\sigma}(\boldsymbol{u}, p): \nabla \boldsymbol{v}_{f} \mathrm{~d} \boldsymbol{x}-\int_{\Gamma_{\mathrm{in}-\mathrm{out}}} \boldsymbol{g} \cdot \boldsymbol{v}_{f} \mathrm{~d} \boldsymbol{a}-\int_{\Omega_{f}\left(\widehat{\boldsymbol{d}_{f}}\right)} q \operatorname{div} \boldsymbol{u} \mathrm{d} \boldsymbol{x} \\
+\int_{\widehat{\Omega}_{s}} \rho_{0} \frac{\partial^{2} \widehat{\boldsymbol{d}_{s}}}{\partial t^{2}} \cdot \widehat{\boldsymbol{v}_{s}} \mathrm{~d} \hat{\boldsymbol{x}}+\int_{\widehat{\Omega}_{s}} \frac{\partial W}{\partial F}\left(\mathrm{I}+\nabla \widehat{\boldsymbol{d}_{s}}\right): \nabla \widehat{\boldsymbol{v}_{s}} \mathrm{~d} \hat{\boldsymbol{x}}=0,
\end{array}
$$

for all $\left(\widehat{\boldsymbol{v}_{f}}, \widehat{q}\right) \in\left[H^{1}\left(\widehat{\Omega}_{f}\right)\right]^{3} \times L^{2}\left(\widehat{\Omega}_{f}\right)$ and $\widehat{\boldsymbol{v}}_{s} \in\left[H_{\Gamma_{D}}^{1}\left(\widehat{\Omega}_{s}\right)\right]^{3}$ with $\widehat{\boldsymbol{v}_{f}}=\widehat{\boldsymbol{v}_{s}}$ on $\widehat{\Sigma}$. The weak form of the geometry coupling conditions [13] and [14] are rewritten in terms of the interface displacement $\gamma \in\left[H^{\frac{1}{2}}(\widehat{\Sigma})\right]^{3}$ as

$$
\int_{\widehat{\Omega}_{f}}\left(\widehat{\boldsymbol{d}_{f}}-\operatorname{Ext}(\gamma)\right) \cdot \widehat{\boldsymbol{\tau}} \mathrm{d} \hat{\boldsymbol{x}}+\int_{\widehat{\Sigma}}\left(\widehat{\boldsymbol{d}_{s}}-\gamma\right) \cdot \widehat{\boldsymbol{\zeta}} \mathrm{d} \hat{\boldsymbol{a}}=0
$$


for all $\widehat{\tau} \in\left[L^{2}\left(\widehat{\Omega}_{f}\right)\right]^{3}$ and $\widehat{\zeta} \in\left[L^{2}(\widehat{\Sigma})\right]^{3}$. Finally, the continuity of the velocities at the interface [15] is reformulated as

$$
\int_{\widehat{\Sigma}}\left(\widehat{\boldsymbol{u}}-\widehat{\boldsymbol{w}}\left(\widehat{\boldsymbol{d}_{f}}\right)\right) \cdot \widehat{\boldsymbol{\xi}} \mathrm{d} \hat{\boldsymbol{a}}=0
$$

for all $\widehat{\boldsymbol{\xi}} \in\left[L^{2}(\widehat{\Sigma})\right]^{3}$.

The weak formulation [18] follows from the summation of [32]-[34].

\section{B. Weak state operator derivatives}

The linearized fluid operator at state $\left(\widehat{\boldsymbol{u}}, \widehat{p}, \widehat{\boldsymbol{d}}_{f}, \gamma\right) \in\left[H^{1}\left(\widehat{\Omega}_{f}\right)\right]^{3} \times L^{2}\left(\widehat{\Omega}_{f}\right) \times$ $\left[H^{1}\left(\widehat{\Omega}_{f}\right)\right]^{3} \times\left[H^{\frac{1}{2}}(\widehat{\Sigma})\right]^{3}$ is denoted by

$$
\begin{array}{r}
\operatorname{DF}\left(\widehat{\boldsymbol{u}}, \widehat{p}, \widehat{\boldsymbol{d}}_{f}, \boldsymbol{\gamma}\right):\left[H^{1}\left(\widehat{\Omega}_{f}\right)\right]^{3} \times L^{2}\left(\widehat{\Omega}_{f}\right) \times\left[H^{1}\left(\widehat{\Omega}_{f}\right)\right]^{3} \times\left[H^{\frac{1}{2}}(\widehat{\Sigma})\right]^{3} \longrightarrow \\
\left(\left[H_{\widehat{\Sigma}}^{1}\left(\widehat{\Omega}_{f}\right)\right]^{3} \times L^{2}\left(\widehat{\Omega}_{f}\right) \times\left[L^{2}(\widehat{\Sigma})\right]^{3} \times\left[L^{2}\left(\widehat{\Omega}_{f}\right)\right]^{3}\right)^{\prime},
\end{array}
$$

and is given by

$$
\begin{aligned}
& \left\langle\mathrm{D} \mathcal{F}\left(\widehat{\boldsymbol{u}}, \widehat{p}, \widehat{\boldsymbol{d}_{f}}, \boldsymbol{\gamma}\right) \cdot\left(\delta \widehat{\boldsymbol{u}}, \delta \widehat{p}, \delta \widehat{\boldsymbol{d}_{f}}, \delta \boldsymbol{\gamma}\right),\left(\widehat{\boldsymbol{v}_{f}}, \widehat{q}, \widehat{\boldsymbol{\xi}}, \widehat{\boldsymbol{\tau}}\right)\right\rangle \\
= & \int_{\Omega_{F}\left(\widehat{\boldsymbol{d}_{f}}\right)} \operatorname{div}\left[\rho_{f} \delta \boldsymbol{u} \otimes\left(\boldsymbol{u}^{n}-\boldsymbol{w}\left(\widehat{\boldsymbol{d}_{f}}\right)\right)\right] \cdot \boldsymbol{v}_{f} \mathrm{~d} \boldsymbol{x} \\
& +\int_{\Omega_{F}\left(\widehat{\boldsymbol{d}_{f}}\right)} \boldsymbol{\sigma}(\delta \boldsymbol{u}, \delta p): \nabla \boldsymbol{v}_{f} \mathrm{~d} \boldsymbol{x} \\
- & \int_{\Omega_{F}\left(\widehat{\boldsymbol{d}_{f}}\right)} q \operatorname{div} \delta \boldsymbol{u} \mathrm{d} \boldsymbol{x}+\frac{1}{\delta t} \int_{\Omega_{F}\left(\widehat{\boldsymbol{d}_{f}}\right)}\left(\operatorname{div} \delta \widehat{\boldsymbol{d}_{f}}\right) \rho_{f} \boldsymbol{u} \cdot \boldsymbol{v}_{f} \mathrm{~d} \boldsymbol{x} \\
+ & \int_{\Omega_{F}\left(\widehat{\boldsymbol{d}_{f}}\right)} \operatorname{div}\left\{\rho_{f} \boldsymbol{u} \otimes\left(\boldsymbol{u}^{n}-\boldsymbol{w}\left(\widehat{\boldsymbol{d}_{f}}\right)\right)\left[\mathrm{I} \operatorname{div} \delta \widehat{\boldsymbol{d}_{f}}-\left(\nabla \delta \widehat{\boldsymbol{d}_{f}}\right)^{\mathrm{T}}\right]\right\} \cdot \boldsymbol{v}_{f} \mathrm{~d} \boldsymbol{x} \\
- & \frac{1}{\delta t} \int_{\Omega_{F}\left(\widehat{\boldsymbol{d}_{f}}\right)} \operatorname{div}\left(\rho_{f} \boldsymbol{u} \otimes \delta \widehat{\boldsymbol{d}_{f}}\right) \cdot \boldsymbol{v}_{f} \mathrm{~d} \boldsymbol{x} \\
& +\int_{\Omega_{F}\left(\widehat{\boldsymbol{d}_{f}}\right)} \boldsymbol{\sigma}(\boldsymbol{u}, p)\left[\mathrm{I} \operatorname{div} \delta \widehat{\boldsymbol{d}_{f}}-\left(\nabla \delta \widehat{\boldsymbol{d}_{f}}\right)^{\mathrm{T}}\right]: \nabla \boldsymbol{v}_{f} \mathrm{~d} \boldsymbol{x} \\
- & \int_{\Omega_{F}\left(\widehat{\boldsymbol{d}_{f}}\right)} \mu\left[\nabla \boldsymbol{u} \nabla \delta \widehat{\boldsymbol{d}_{f}}+\left(\nabla \delta \widehat{\boldsymbol{d}_{f}}\right)^{\mathrm{T}}(\nabla \boldsymbol{u})^{\mathrm{T}}\right]: \nabla \boldsymbol{v}_{f} \mathrm{~d} \boldsymbol{x} \\
- & \int_{\Omega_{F}\left(\widehat{\boldsymbol{d}_{f}}\right)} q \operatorname{div}\left\{\boldsymbol{u}\left[\mathrm{I} \operatorname{div} \delta \widehat{\boldsymbol{d}_{f}}-\left(\nabla \delta \widehat{\boldsymbol{d}_{f}}\right)^{\mathrm{T}}\right]\right\} \mathrm{d} \boldsymbol{x}+\int_{\widehat{\Sigma}}\left(\delta \widehat{\boldsymbol{u}}-\frac{\delta \widehat{\boldsymbol{d}_{f}}}{\delta t}\right) \cdot \widehat{\boldsymbol{\xi}} \mathrm{d} \hat{\boldsymbol{a}} \\
+ & \frac{\rho}{\delta t} \int_{\Omega_{F}\left(\widehat{\boldsymbol{d}_{f}}\right)} \delta \boldsymbol{u} \cdot \boldsymbol{v}_{f} \mathrm{~d} \boldsymbol{x}+\int_{\widehat{\Omega_{F}}}\left(\delta \widehat{\boldsymbol{d}_{f}}-\operatorname{Ext}(\delta \gamma)\right) \cdot \widehat{\boldsymbol{\tau}} \mathrm{d} \hat{\boldsymbol{x}}
\end{aligned}
$$


for all $\left(\widehat{\boldsymbol{v}_{f}}, \widehat{q}, \widehat{\boldsymbol{\xi}}, \widehat{\boldsymbol{\tau}}\right) \in\left[H^{1}\left(\widehat{\Omega}_{f}\right)\right]^{3} \times L^{2}\left(\widehat{\Omega}_{f}\right) \times\left[L^{2}(\widehat{\Sigma})\right]^{3} \times\left[L^{2}\left(\widehat{\Omega}_{f}\right)\right]^{3}$.

The linearized solid operator at state $\left(\widehat{\boldsymbol{d}}_{s}, \boldsymbol{\gamma}\right) \in\left[H_{\Gamma_{D}}^{1}\left(\widehat{\Omega}_{s}\right)\right]^{3} \times\left[L^{2}(\widehat{\Sigma})\right]^{3}$

$$
\mathrm{D} \mathcal{S}\left(\widehat{\boldsymbol{d}}_{s}, \boldsymbol{\gamma}\right):\left[H_{\Gamma_{D}}^{1}\left(\widehat{\Omega}_{s}\right)\right]^{3} \times\left[H^{\frac{1}{2}}(\widehat{\Sigma})\right]^{3} \longrightarrow\left(\left[H_{\Gamma_{D} \cup \widehat{\Sigma}}^{1}\left(\widehat{\Omega}_{s}\right)\right]^{3} \times\left[L^{2}(\widehat{\Sigma})\right]^{3}\right)^{\prime},
$$

is given by

$$
\begin{aligned}
& \left\langle\mathrm{D} \mathcal{S}\left(\widehat{\boldsymbol{d}}_{s}, \boldsymbol{\gamma}\right) \cdot\left(\delta \widehat{\boldsymbol{d}_{s}}, \delta \boldsymbol{\gamma}\right),\left(\widehat{\boldsymbol{v}_{s}}, \widehat{\boldsymbol{\zeta}}\right)\right\rangle=\frac{2}{(\delta t)^{2}} \int_{\widehat{\Omega}_{S}} \rho_{0} \delta \widehat{\boldsymbol{d}_{s}} \cdot \boldsymbol{v}_{s} \mathrm{~d} \hat{\boldsymbol{x}} \\
& \quad+\frac{1}{2} \int_{\widehat{\Omega}_{S}} \nabla \delta \widehat{\boldsymbol{d}_{s}}:\left(\frac{\partial^{2} W}{\partial F^{2}}\left(I+\nabla \widehat{\boldsymbol{d}_{s}}\right)\right): \nabla \boldsymbol{v}_{s} \mathrm{~d} \hat{\boldsymbol{x}}+\int_{\widehat{\Sigma}}\left(\delta \widehat{\boldsymbol{d}_{s}}-\delta \gamma\right) \cdot \widehat{\boldsymbol{\zeta}} \mathrm{d} \hat{\boldsymbol{a}},
\end{aligned}
$$

for all $\left(\widehat{\boldsymbol{v}}_{s}, \widehat{\boldsymbol{\zeta}}\right) \in\left[H_{\Gamma_{D}}^{1}\left(\widehat{\Omega}_{s}\right)\right]^{3} \times\left[L^{2}(\widehat{\Sigma})\right]^{3}$.

We finally introduce the linearized interface operator at state $\left(\widehat{\boldsymbol{u}}, \widehat{p}, \widehat{\boldsymbol{d}}_{f}, \widehat{\boldsymbol{d}}_{s}\right)$

$\mathrm{D} \mathcal{I}\left(\widehat{\boldsymbol{u}}, \widehat{p}, \widehat{\boldsymbol{d}}_{f}, \widehat{\boldsymbol{d}}_{s}\right):\left[H^{1}\left(\widehat{\Omega}_{f}\right)\right]^{3} \times L^{2}\left(\widehat{\Omega}_{f}\right) \times\left[H^{1}\left(\widehat{\Omega}_{f}\right)\right]^{3} \times\left[H^{1}\left(\widehat{\Omega}_{s}\right)\right]^{3} \longrightarrow\left[H^{-\frac{1}{2}}(\widehat{\Sigma})\right]^{3}$, defined by

$$
\begin{array}{r}
\left\langle\mathrm{DI}\left(\widehat{\boldsymbol{u}}, \widehat{p}, \widehat{\boldsymbol{d}}_{f}, \widehat{\boldsymbol{d}}_{s}\right) \cdot\left(\delta \widehat{\boldsymbol{u}}, \delta \widehat{p}, \delta \widehat{\boldsymbol{d}}_{f}, \delta \widehat{\boldsymbol{d}}_{s}\right), \boldsymbol{\mu}\right\rangle \\
=\left\langle\mathrm{DF}\left(\widehat{\boldsymbol{u}}, \widehat{p}, \widehat{\boldsymbol{d}}_{f}, \mathbf{0}\right) \cdot\left(\delta \widehat{\boldsymbol{u}}, \delta \widehat{p}, \delta \widehat{\boldsymbol{d}}_{f}, \mathbf{0}\right),\left(\mathcal{L}_{f} \boldsymbol{\mu}, 0, \mathbf{0}, \mathbf{0}\right)\right\rangle \\
+\left\langle\mathrm{D} \mathcal{S}\left(\widehat{\boldsymbol{d}}_{s}, \mathbf{0}\right)\left(\delta \widehat{\boldsymbol{d}}_{s}, \mathbf{0}\right),\left(\mathcal{L}_{s} \boldsymbol{\mu}, \mathbf{0}\right)\right\rangle,
\end{array}
$$

for all $\boldsymbol{\mu} \in\left[H^{\frac{1}{2}}(\widehat{\Sigma})\right]^{3}$. 